\title{
Glutamine Is Required for Persistent Epileptiform Activity in the Disinhibited Neocortical Brain Slice
}

\author{
Hiroaki Tani, Chris G. Dulla, John R. Huguenard, and Richard J. Reimer \\ Department of Neurology and Neurological Sciences, Stanford University School of Medicine, Stanford, California 94305
}

The neurotransmitter glutamate is recycled through an astrocytic-neuronal glutamate-glutamine cycle in which synaptic glutamate is taken up by astrocytes, metabolized to glutamine, and transferred to neurons for conversion back to glutamate and subsequent release. The extent to which neuronal glutamate release is dependent upon this pathway remains unclear. Here we provide electrophysiological and biochemical evidence that in acutely disinhibited rat neocortical slices, robust release of glutamate during sustained epileptiform activity requires that neurons be provided a continuous source of glutamine. We demonstrate that the uptake of glutamine into neurons for synthesis of glutamate destined for synaptic release is not strongly dependent on the system A transporters, but requires another unidentified glutamine transporter or transporters. Finally, we find that the attenuation of network activity through inhibition of neuronal glutamine transport is associated with reduced frequency and amplitude of spontaneous events detected at the single-cell level. These results indicate that availability of glutamine influences neuronal release of glutamate during periods of intense network activity.

\section{Introduction}

While presynaptic reuptake systems recycle most neurotransmitters, $\sim 70 \%$ of released glutamate is recycled through an astrocytic-neuronal glutamate-glutamine cycle (Lieth et al., 2001; Sibson et al., 2001). In this pathway (Fig. 1), astrocytes take up and metabolize synaptically released glutamate to glutamine, which is transferred to neurons for conversion back to glutamate (Broman et al., 2000). Molecular segregation establishes directional flow: glutamate release mechanisms are confined to presynaptic neurons; astrocytic transporters clear released glutamate from the synapse; and glutamine synthetase and phosphate-activated glutaminase, the primary metabolic enzymes of the cycle, are expressed in astrocytes and neurons, respectively (Kvamme, 1998; Kaneko, 2000; Danbolt, 2001). Glutamate can also be derived from other sources, most significantly the TCA cycle intermediate $\alpha$-ketoglutarate (Kvamme, 1998; McKenna, 2007). Net synthesis of TCA cycle intermediates (anaplerosis) from glucose, however, requires pyruvate carboxylase, an enzyme expressed in astrocytes, but not neurons (Shank et al., 1985). Thus, glutamate derived from glucose is produced predominantly in astrocytes and must be metabolized by glutamine synthetase and transit through part of the cycle before contributing to the neurotransmitter pool.

Received Jan. 8, 2009; revised Dec. 1, 2009; accepted Dec. 5, 2009.

This work was supported by the National Institutes of Health [Grants NS045634 (R.J.R.), NS012151 (J.R.H.), and NS007280 (C.G.D.)], a Dana Foundation Brain Immuno-Imaging Grant (R.J.R.), and fellowships from the American Epilepsy Foundation (H.T., C.G.D.). We thank Trent Anderson for assistance with electrophysiology experiments.

Correspondence should be addressed to Richard J. Reimer, Department of Neurology and Neurological Sciences, Stanford University School of Medicine, P211 MSLS 1201 Welch Road, Stanford, CA 94305. E-mail: rjreimer@stanford.edu.

DOI:10.1523/JNEUROSCI.0106-09.2010

Copyright $\odot 2010$ the authors $\quad 0270-6474 / 10 / 301288-13 \$ 15.00 / 0$
Due to the complex multicellular nature of the cycle, much of what is known about its role comes from studies in living animals and humans: radiotracer and NMR studies have demonstrated that the majority of synaptic glutamate is derived from the cycle (Kvamme, 1998; Rothman et al., 2003), and pharmacological and genetic manipulations have demonstrated that blocking the cycle causes behavioral deficits (Gibbs et al., 1996; Masson et al., 2006). Acutely isolated brain slices should be a useful adjunct-they retain complex anatomical and functional intercellular connectivity, but permit pharmacological manipulation and synaptic level analysis with electrophysiology. Although slice studies have demonstrated that glutamine influences glutamate levels (Kapetanovic et al., 1993; Rae et al., 2003), direct analyses of synaptic transmission with the glutamate-glutamine cycle disrupted have failed to uncover marked effects (Masson et al., 2006; Kam and Nicoll, 2007). This may be explained, in part, by increased de novo synthesis of glutamate in response to the demands of neuronal activity (Oz et al., 2004; Henry et al., 2007).

To address discrepancies between in vivo and slice studies, we took advantage of the increased demand on glutamate synthetic pathways during epileptiform activity (Bacci et al., 2002; Otsuki et al., 2005; Giove et al., 2006; Tani et al., 2007) to create a dependence on the cycle. Using pharmacologically disinhibited neocortex, a simple model in which electrically evoked activity can be modulated by the intensity of the stimulus (Courtney and Prince, 1977; Gutnick et al., 1982) and epileptiform events can be analyzed as a readout of neuronal activity (Aram and Lodge, 1988), we demonstrate that synthesis of glutamine in astrocytes and its transfer to neurons are essential for sustained excitatory neuronal activity. We also show that uptake of glutamine into neurons for synthesis of glutamate is not dependent on system A transporters, but requires another unidentified glutamine transporter, and that blocking glutamine transport reduces 


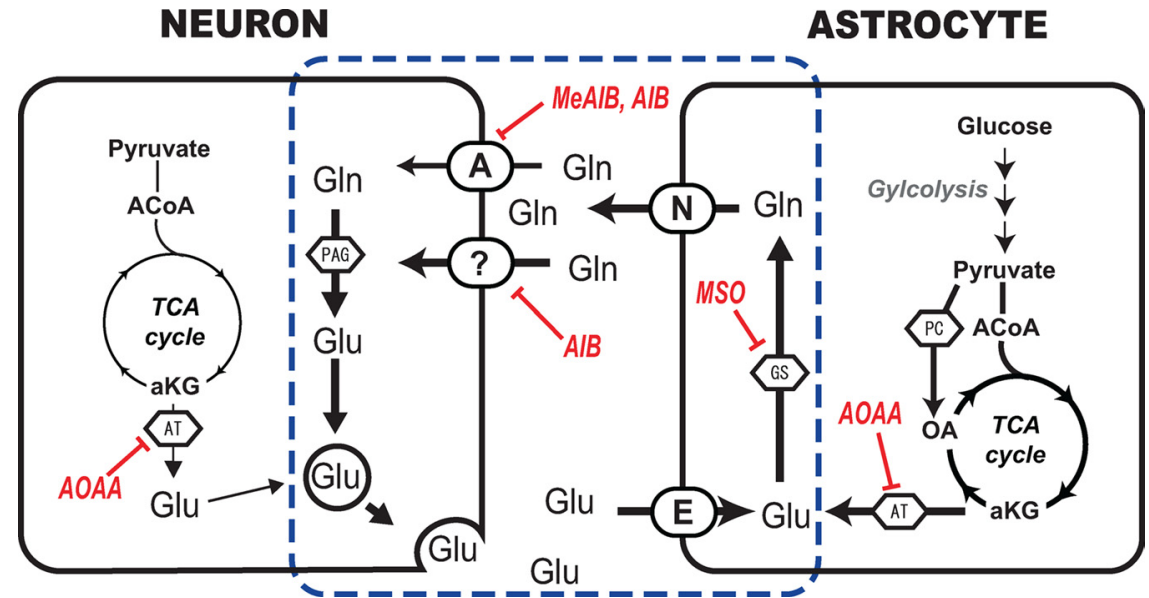

Figure 1. Synthesis and metabolism of synaptically released glutamate. In the glutamate-glutamine shuttle (outlined in dashed blue line), released glutamate is taken up by astrocytes and metabolized to glutamine, which is then transferred back to neurons. The transmitter is cleared from the synapse by astrocytic excitatory amino acid transporters (EAATs; E), and GS in astrocytes rapidly metabolizes glutamate to glutamine. Efflux of glutamine from astrocytes is thought to be mediated by the system N transporters SNAT3 and SNAT 5 (N), while the system A transporters SNAT1 and SNAT2 (A) are thought to mediate neuronal uptake of glutamine, but other unidentified non-system A glutamine transporters (?) may also contribute. Within neurons, phosphate-activated glutaminase (PAG) resynthesizes glutamate from glutamine to complete the cycle. Glutamate can also be synthesized from glucose in astrocytes through the conversion of the TCA cycle intermediate $\alpha$-ketoglutarate by the activity of amino transferases (AT). Net synthesis of TCA cycle intermediates from glucose requires pyruvate carboxylase (PC), an enzyme expressed in astrocytes, but not detected in neurons. For glucose-derived glutamate to contribute to the neurotransmitter pool, it must, therefore, enter the cycle at the level of $G S$ and be transferred to neurons as a glutamine intermediate. Inhibitors in this study are in indicated in red (MeAIB inhibits system A transporters; AIB is a nonspecific inhibitor of glutamine transporters; AOAA inhibits amino transferases; MSO inhibits glutamine synthetase).

frequency and amplitude of spontaneous events, suggesting that glutamine availability influences synaptic glutamate release during periods of intense activity.

\section{Materials and Methods}

Preparation of brain slices. Briefly, following all guidelines of Stanford University's Institutional Animal Care and Use Committee, Sprague Dawley rats (3-7 weeks) were anesthetized $(55 \mathrm{mg} / \mathrm{kg}$ pentobarbital through intraperitoneal injection) and decapitated, and the brains were rapidly removed and placed in chilled $\left(4^{\circ} \mathrm{C}\right)$ low- $-\mathrm{Ca}^{2+}$, low- $-\mathrm{Na}^{+}$slicing solution consisting of the following (in $\mathrm{mM}$ ): 234 sucrose, 11 glucose, 24 $\mathrm{NaHCO}_{3}, 2.5 \mathrm{KCl}, 1.25 \mathrm{NaH}_{2} \mathrm{PO}_{4}, 10 \mathrm{MgSO}_{4}$, and $0.5 \mathrm{CaCl}_{2}$, equilibrated with a mixture of $95 \% \mathrm{O}_{2}$ and $5 \% \mathrm{CO}_{2}$. The brain was glued to the slicing stage of a Leica VT1200 sectioning system and slices $(400 \mu \mathrm{m}$ for field recordings or $300 \mu \mathrm{m}$ for whole-cell recordings) were cut in a coronal orientation. The slices were then incubated for $1 \mathrm{~h}$ in $32^{\circ} \mathrm{C}$ oxygenated artificial CSF (aCSF) consisting of the following (in mM): $126 \mathrm{NaCl}$, $26 \mathrm{NaHCO}_{3}, 3 \mathrm{KCl}, 1.25 \mathrm{NaH}_{2} \mathrm{PO}_{4}, 2 \mathrm{MgCl}_{2}, 2 \mathrm{CaCl}_{2}$, and 10 glucose.

Field recordings. Slices were placed in an interface chamber maintained at $34^{\circ} \mathrm{C}$ and superfused with oxygenated aCSF at $2 \mathrm{ml} / \mathrm{min}$ and stimulated with a concentric bipolar electrode at layer VI-white matter boundary with $300-500 \mu \mathrm{A}, 500 \mu$ s pulses at $5 \mathrm{~s}$ intervals (unless otherwise indicated) delivered by a stimulus isolator (World Precision Instruments). Electrophysiological data were recorded from glass micropipettes electrodes $\sim 1 \mathrm{M} \Omega$ filled with aCSF using an Axon Multiclamp $700 \mathrm{~A}$ amplifier and Digidata 1322A digitizer with pClamp software (Molecular Devices).

Whole-cell recordings. Slices were placed in submerged chamber maintained at $34^{\circ} \mathrm{C}$ and superfused with oxygenated aCSF at $2 \mathrm{ml} / \mathrm{min}$ and stimuli were applied as described in the Field recordings section above. Electrophysiological voltage-clamp data were obtained from glass micropipettes electrodes $\sim 3 \mathrm{M} \Omega$ filled with a low-chloride internal solution $\left(E_{\mathrm{Cl}}=E_{\text {holding }}=-60 \mathrm{mV}\right)$ consisting of (in $\mathrm{mm}$ ) $130 \mathrm{~K}$-gluconate, 10 $\mathrm{KCl}, 2 \mathrm{NaCl}, 10 \mathrm{HEPES}$, and $10 \mathrm{EGTA}$. Spontaneous activity was analyzed using custom software (wdectecta).
Biosensor imaging. Collection and analysis of glutamate biosensor data was done as previously described (Dulla et al., 2008). Briefly, a 50 $\mu \mathrm{l}$ aliquot of purified bacterially expressed FLII81E-1 $\mu \mathrm{M}$ FRET-based glutamate biosensor was applied to the surface of slices in a modified interface incubation chamber for 10-20 min. Slices were then placed into the recording chamber of an Axioskop microscope (Carl Zeiss) with continual superfusion of aCSF for simultaneous imaging with a Zeiss $2.5 \times$ Fluar objective and electrophysiological recording. Excitation with $440 \mathrm{~nm}$ wavelength light was used with computer control of shutter opening and closing (3-6 s illumination/exposure). Each imaging experiment consisted of five exposures with a $5 \mathrm{~s}$ interstimulus interval (ISI) and five additional exposures at 2.5, 15, or $30 \mathrm{~s}$ ISI. A Neuro-CCD camera (RedShirtImaging) was used to collect imaging data at $500 \mathrm{~Hz}$. Emission signals first passed through a 455 DCLP dichroic mirror to eliminate excitation fluorescence and then separated into two channels using a Photometrics Dual-View two channel imaging system to isolate CFP and Venus signals. Ratiometric images of CFP/Venus signals were then created and further analyzed. Pixels with glutamate biosensor ratio values of 0.65 or greater were considered to be above threshold.

Amino acid analysis. After field recording experiments, cortical slices were removed from the rig and washed three times in ice-cold aCSF. The hippocampi and other noncortical structures were removed, and the resulting cortical strips were placed in $400 \mu \mathrm{l}$ of heated $\left(90^{\circ} \mathrm{C}\right) 75 \%$ ethanol in a screw-capped tube, shaken vigorously, and then incubated for $10 \mathrm{~min}$ at $90^{\circ} \mathrm{C}$ followed by transfer to ice. After centrifugation at $10,000 \times g$ for $10 \mathrm{~min}$ the supernatant was removed and lyophilized, resuspended in $100 \mu \mathrm{l}$ of $150 \mathrm{~mm} \mathrm{NaCl}$, and submitted to the Stanford University Mass Spectrometry facility for amino acid analysis.

Radiotracer studies. To generate cortical slices for radiotracer studies a vertical scalpel incision was made on a block-mounted rat brain at the midline followed by a transsagittal cut. Coronal slices (350 $\mu \mathrm{m}$ thick) were trimmed in ice-cold slicing chamber to remove the hippocampus and other noncortical structures and transferred to oxygenated aCSF in a $32^{\circ} \mathrm{C}$ for $1 \mathrm{~h}$. Slices were then transferred to oxygenated nominally $\mathrm{Mg}^{2+}$-free aCSF and incubated for $30 \mathrm{~min}$ at room temperature. Individual slices were then transferred with a small spatula to $1.5 \mathrm{ml}$ conical tubes containing $1 \mathrm{ml}$ of oxygenated $\mathrm{Mg}^{2+}$-free aCSF with L-[2,3,4${ }^{3} \mathrm{H}$ ]glutamine $(1 \mu \mathrm{Ci}), 200 \mu \mathrm{M}$ TBOA, and indicated concentrations of aminoisobutyric acid (AIB) or methylaminoisobutyric acid (MeAIB) and then incubated at $32^{\circ} \mathrm{C}$ for the specified times. To terminate the reaction slices were washed three times in ice-cold aCSF and then placed in $400 \mu \mathrm{l}$ of $75 \%$ ethanol in a screw cap tube preheated to $90^{\circ} \mathrm{C}$, shaken vigorously, incubated for $1 \mathrm{~min}$ at $90^{\circ} \mathrm{C}$, and transferred to ice. To determine glutamine uptake, aliquots of the ethanol homogenate were diluted in scintillation fluid (CytoScint, ICN) for analysis on Beckman LS6000SC gamma counter. To measure production of $\left[{ }^{3} \mathrm{H}\right]$ glutamate, after centrifugation to remove insoluble material, the glutamate fraction of the ethanol extract was isolated by ion exchange chromatography (Darmaun et al., 1985). Briefly, ion exchange columns (Biorad AG 1-X8 formate resin) were equilibrated with $0.1 \mathrm{~m}$ borate buffer, $\mathrm{pH}$ 9.0, and the samples were applied. After extensive washing with the borate buffer, glutamate was eluted using $1 \mathrm{~m}$ acetic acid. To measure glutamate release, slices were incubated with $\left[{ }^{3} \mathrm{H}\right]$ glutamine and TBOA with or without AIB or MeAIB for $30 \mathrm{~min}$, washed three times in $\mathrm{Mg}^{2+}$-free aCSF, and then incubated in $\mathrm{Mg}^{2+}$-free aCSF with TBOA with or without AIB or MeAIB for $20 \mathrm{~min}$, at which time the incubation media was removed and subjected to ion 
A

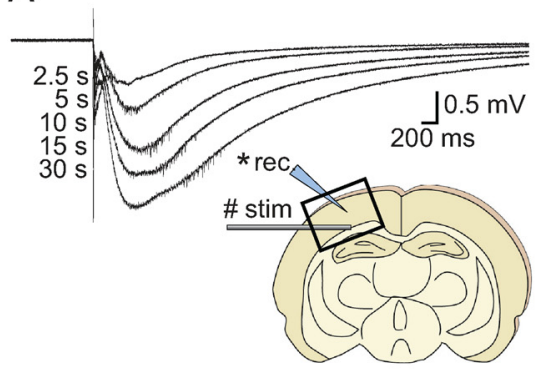

$\mathrm{B}$

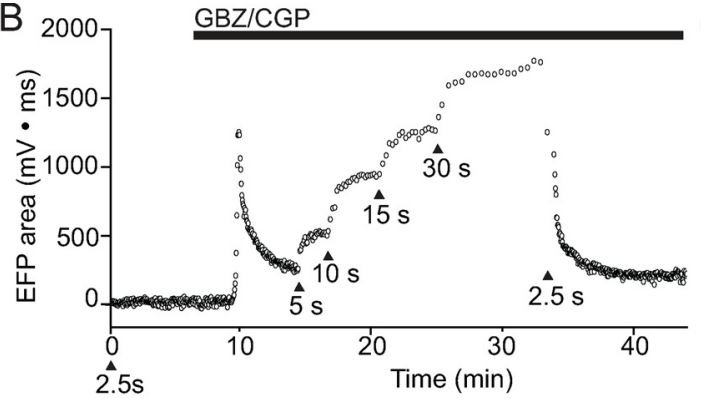

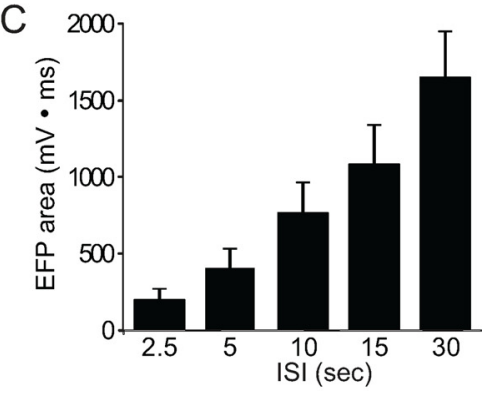
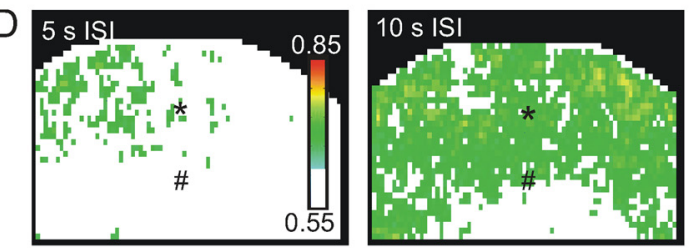

E
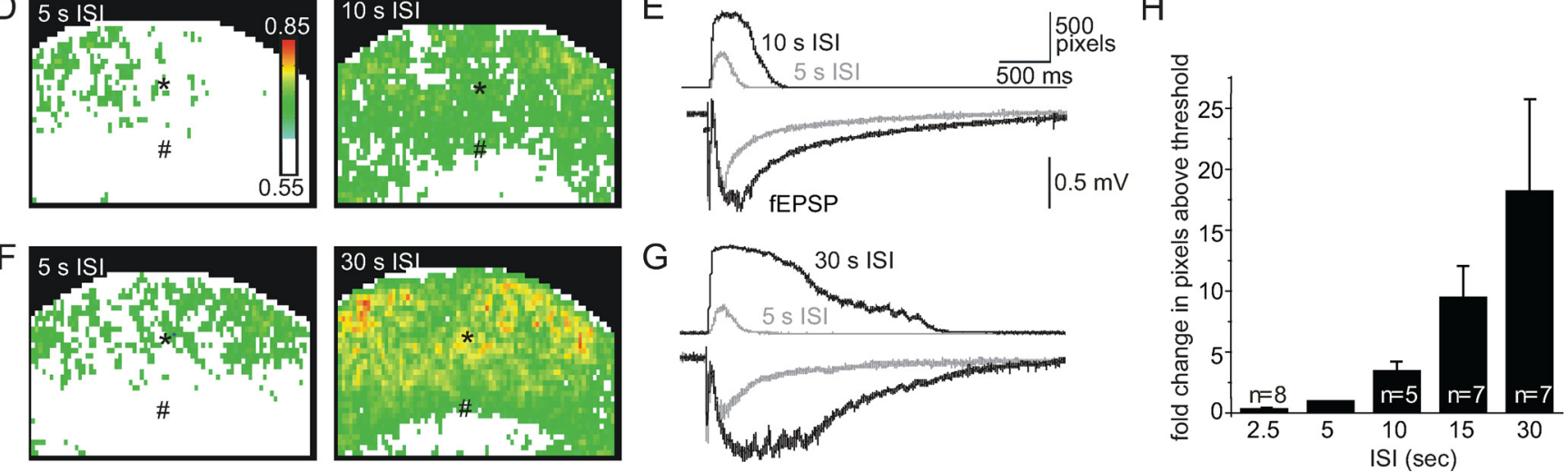

Figure 2. Evoked field potential size and glutamate release correlate directly with interstimulus interval in pharmacologically disinhibited neocortical slices. $A$, Representative traces of EFPs recorded from an electrode (rec) in layer V in a GBZ/GGP-treated slice with a stimulating electrode (stim) placed at the layer VI-white matter interface at ISIs of 2.5, 5 10, 15, and 30 s demonstrate qualitatively larger fields with longer ISIs. B, A plot of evoked field potential area (in millivolt • milliseconds) over time from a representative experiment demonstrates rapid changes in evoked potential area with incremental increases in ISI. Arrowheads indicate time at which the ISI was changed. Initial ISI at time 0 was $2.5 \mathrm{~s}$. At $\sim 7$ min into the recording, the GBZ/CGP was added to the superfusion solution and at $10 \mathrm{~min}$ epileptiform responses ensued as seen as a transient increase in EFP amplitude to near $1500 \mathrm{mV} \cdot \mathrm{ms}$ and a subsequent decay to $<500 \mathrm{mV} \cdot \mathrm{ms}$. At $14 \mathrm{~min}$, the ISI was switched to $5 \mathrm{~s}$, and the responses increased in amplitude, as also occurred with each subsequent increase in ISI. Restoration to the original ISI at 34 min resulted in reduction of EFPs to original 2.5 s levels. C, Evoked field potential area measured in several slices over the range of ISIs demonstrates consistency of ISI effect on EFPs $(F=146.09 ; p<0.001 ; n=6)$. For each slice, 10 sweeps were averaged $2 \mathrm{~min}$ following each ISI change to allow for stabilization. D, Examples of glutamate biosensor signal changes in a cortical slice at 5 and $10 \mathrm{~s} I S I \mathrm{~s}$ indicate a large increase in the area of cortex recruited into the network with longer ISIs. Pseudo-color scale for the biosensor signal (inverse of the FRET ratio) is shown (inset in $\boldsymbol{D}$ ). The box in the diagram in $\boldsymbol{A}$ approximates the imaged region with the same orientation (pial surface at top) along with the location of stimulating (\#) and recording $\left(^{*}\right)$ electrodes. $E$, Example evoked field potentials (bottom traces) and number of pixels above threshold $(>0.65$ ) for biosensor (top traces) for the two conditions in $\boldsymbol{D}$. Both EFPs and glutamate signal increased as the ISI was increased from $5 \mathrm{~s}$ (gray) to $10 \mathrm{~s}$ (black). $\boldsymbol{F}$, A similar, but more dramatic, change in the glutamate biosensor signal is seen as the ISI is increased from 5 to 30 s. G, A marked prolongation of the evoked field potential (bottom traces) and the glutamate biosensor transient (top traces) is associated with increasing the ISI from $5 \mathrm{~s}$ (gray) to $30 \mathrm{~s}$ (black). $\boldsymbol{H}$, There was a direct correlation between the ISI and the area of cortex in which glutamate release is detected $(F=17.00 ; p<0.0001)$. Due to photobleaching of the sensor, data were collected at $5 \mathrm{~s} I S I$ and one additional ISI only in each slice. Values were normalized to the $5 \mathrm{~s} I S I$ to permit slice-to-slice comparison.

exchange chromatography as above. Fractions of the acetic acid eluate containing glutamate were identified by previous experiments in which $\left[{ }^{3} \mathrm{H}\right]$ glutamate was added to amino acid extracts or incubation media of naive slices. These fractions were diluted in scintillation fluid and analyzed as described above.

Drugs and reagents. All salts and glucose for use in buffers were obtained from Sigma-Aldrich. In addition the following reagents were also obtained from Sigma-Aldrich: MeAIB (M2383), L-methionine sulfoximine (MSO; M5379), glutamate (G1501), and aminooxyacetic acid (AOAA; C13408). L-glutamine, histidine, and alanine were obtained from Alfa Aesar. AIB (A0323) was obtained from TCI America. GABAzine (1262) and CGP54626 (1088) were obtained from Tocris Bioscience. $\left[{ }^{3} \mathrm{H}\right]$ glutamine was obtained from American Radiolabeled Chemicals. The following stock solutions were made at the indicated concentrations in $\mathrm{H}_{2} \mathrm{O}$, frozen in liquid nitrogen, and stored at $-20^{\circ} \mathrm{C}$ : MeAIB, $1 \mathrm{M}$; MSO, 50-100 mM; L-glutamine, $250 \mathrm{~mm}$; alanine, $1 \mathrm{M}$; glutamate, 250 mM; GABAzine, $10 \mathrm{~mm}$; and CGP54626, $100 \mu \mathrm{M}$. All other reagents were dissolved directly in aCSF. All reagents were used at the concentrations indicated. For experiments in which reagents were added to aCSF, unless

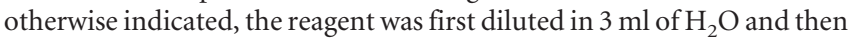
added to $100 \mathrm{ml}$ of aCSF.

To rule out effects of osmolarity changes associated with addition of high mM concentrations of MeAIB and AIB, we tested the effects of hypertonicity by using mannitol at equivalent concentrations $(20-50 \mathrm{~mm})$. This produced only modest effects on evoked field potential amplitude and no effect on probability of evoking an epileptiform field potential (supplemental Fig. 1, available at www.jneurosci.org as supplemental material).

Statistics. Statistical comparisons between two groups were determined using Student's unpaired and paired $t$ test as appropriate. Comparisons between three groups were done using one-way ANOVA followed by Tukey pairwise tests. For each experiment, slices from a minimum of three animals were used.

\section{Results}

Epileptiform responses are directly correlated with extent of glutamate release and are partially refractory

Epileptiform activity can be readily elicited in pharmacologically disinhibited rat neocortical slices (Courtney and Prince, 1977; Aram and Lodge, 1988). To induce disinhibition, we bathed slices with $10 \mu \mathrm{M}$ GABAzine [an inhibitor of $\mathrm{GABA}_{\mathrm{A}}$ receptors (Mienville and Vicini, 1987)] and $10 \mathrm{nM}$ CGP54626 [an inhibitor of $\mathrm{GABA}_{\mathrm{B}}$ receptors (Brugger et al., 1993)]; together, these are referred to as GBZ/CGP. Electrical stimulation of layer VI/white matter boundary in GBZ/CGP-treated slices evoked field potentials recorded in the same column, directly above, in layer V (Fig. $2 A$, inset) that were prolonged and polyphasic. The probability of 

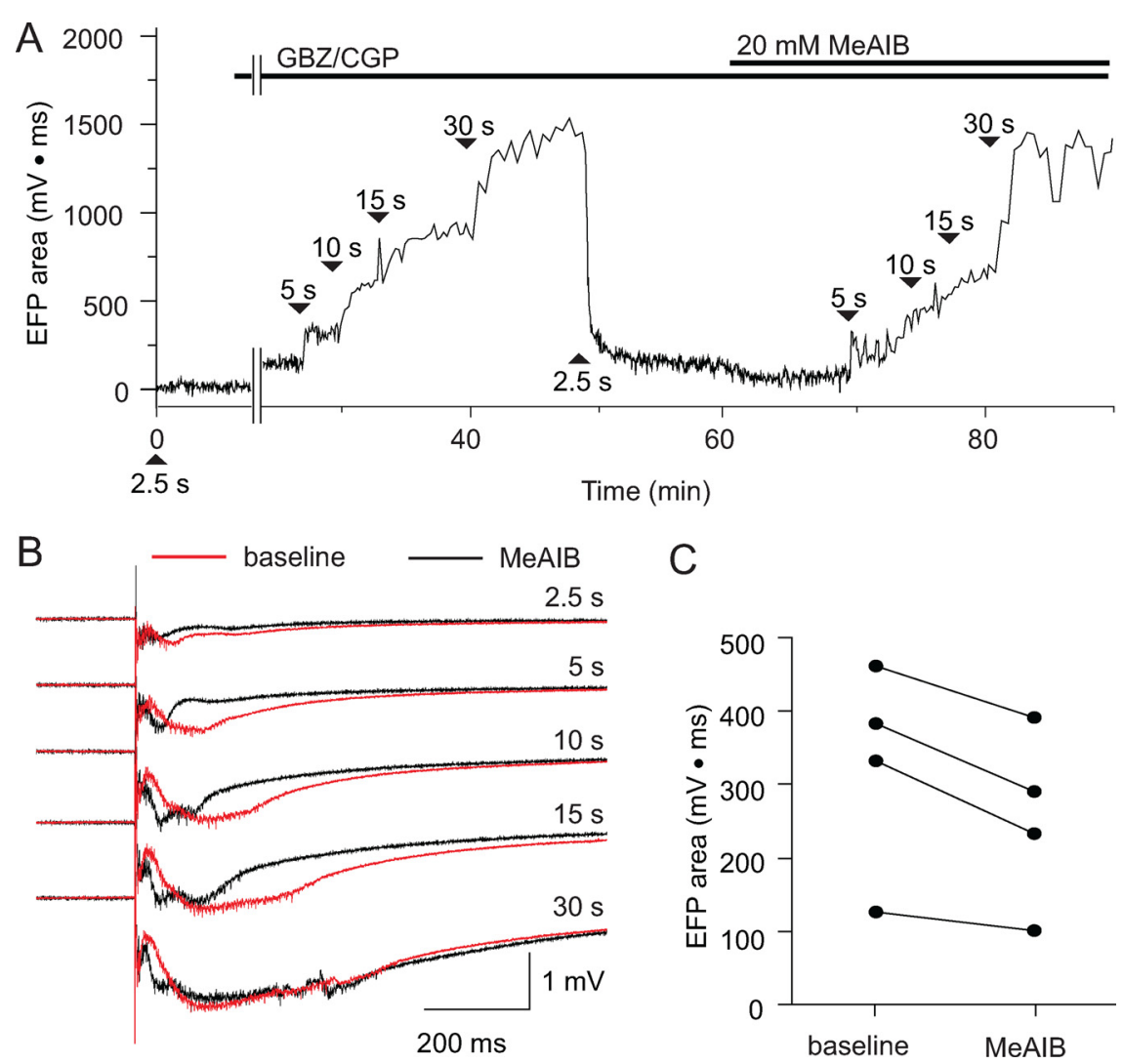

Figure 3. The system A transport system inhibitor MeAIB reduces size, but not probability of epileptiform field potentials. $\boldsymbol{A}$, Time course of evoked field potential area in a GBZ/CGP-treated slice as the ISI is increased from 2.5 to 30 s in the absence and presence of MeAIB demonstrates a small reduction in area associated with addition of $20 \mathrm{~mm}$ MeAIB. Note, however, that large field potentials are robustly elicited (i.e., with every stimulus) in the presence of MeAIB. $\boldsymbol{B}$, Representative traces in the absence (red) and presence (black) of MeAIB at ISIs from 2.5 to 30 s illustrate the effect of MeAIB. Tracings were collected beginning 2 min following ISI change to allow for stabilization. C, Paired analyses of total area at $5 \mathrm{sISI}$ before and $10 \mathrm{~min}$ after the addition of $20 \mathrm{~mm}$ MeAIB show a small but consistent reduction in evoked field potential area $(23.3 \pm 4.8 \% ; n=4 ; p<0.03)$.

inducing such epileptiform field potentials (EFPs) depended on the strength of the electrical stimulus. Near-threshold stimuli failed to uniformly elicit polyphasic EFPs; intermingled failures and success were commonly present. With increased stimulus strength (either duration and/or amplitude), however, EFPs were evoked with nearly every stimulus. After a short stabilization period at suprathreshold intensities, robust EFPs were elicited that were remarkably similar in amplitude and duration for a given ISI. Varying the ISI led to EFPs with amplitudes that directly correlated with ISI (Fig. 2A). Plotting the evoked field areas (in $\mathrm{mV} \cdot \mathrm{ms}$ ) over time demonstrated a large increase in field area soon after wash in of GBZ/CGP and then a decrease to a plateau within minutes (Fig. $2 B$ ). Subsequent stepwise increases in ISI from 2.5 to $30 \mathrm{~s}$ led in each case to increased evoked field areas. With a return to the initial ISI of $2.5 \mathrm{~s}$, the field area again decreased, but no failures occurred. This pattern was consistent from slice to slice with an approximately eightfold difference in the areas of evoked potentials at 2.5 and $30 \mathrm{~s}$ ISIs (Fig. 2C). This association of briefer and smaller EFPs with shorter ISIs suggests that there is a time-sensitive factor (or factors) limiting the propagation of network activity as suggested by previous studies demonstrating a direct correlation between interstimulus interval and the probability of eliciting an epileptiform discharge in pharmacologically disinhibited cortex (Prince, 1966; Courtney and Prince, 1977).

In the disinhibited cortex, a number of variables might affect the extent of field depolarization and hence the total evoked po- tential area. Glutamate-dependent neuronal network activity, however, is likely the most direct correlate, especially under conditions of disinhibition in which synaptically released glutamate is the only ionotropic neurotransmitter that can participate in the network activation. If true, then changes in EFP area could be used as a surrogate measure of synaptically released glutamate. To determine whether changes in EFP area correspond to changes in glutamate release, we used a recently described FRET-based biosensor to monitor extracellular glutamate levels (Dulla et al., 2008). This FRET-based biosensor is a chimeric protein composed of the bacterial periplasmic glutamate binding protein YbeJ with cyan fluorescent protein (CFP) inserted into the $\mathrm{N}$-terminal region of the protein and the yellow fluorescent protein (YFP) variant Venus attached to the carboxy terminus of the glutamate binding protein (Deuschle et al., 2005). When the purified bacterially produced sensor is applied to slices, evoked release of glutamate can be readily detected in pharmacologically disinhibited slices (Dulla et al., 2008). Characterization of the biosensor-treated slices demonstrated that larger EFPs were associated with longer ISIs, indicating that the biosensor did not grossly alter neocortical physiology (see also Dulla et al., 2008). Analysis of the glutamate-dependent FRET signal in these slices showed that increasing the ISI from 5 to $10 \mathrm{~s}$ resulted in a marked increase in the area of cortex with detectable glutamate signal (Fig. 2D,E). Similar but larger changes were seen in a different slice as the ISI was increased from 5 to $30 \mathrm{~s}$ (Fig. $2 F, G$ ). Since photobleaching of the sensor precluded testing a range of ISIs in a single slice, we examined the signal in each slice at a reference ISI of $5 \mathrm{~s}$ and then a single test ISI of $2.5,10,15$, or $30 \mathrm{~s}$. By normalizing the signal to that detected at the ISI of $5 \mathrm{~s}$, we are able to compare the pixels above threshold over the range of ISIs. This analysis revealed a direct correlation between glutamate biosensor signal and the ISI (Fig. $2 H$ ), supporting the conclusion that EFP area can be used to estimate glutamate release in the disinhibited slice.

\section{Neither glutamine replenishment nor system A-dependent glutamine transport are necessary to maintain epileptiform activity in disinhibited cortical slices}

To begin to determine how different factors might contribute to the relationship between the ISI and the amount of glutamate released, we assessed the effect of different manipulations on EFPs. Since glutamine is an immediate metabolic precursor of glutamate (Hamberger et al., 1979) and is present in CSF at $\sim 0.5$ mM (Lerma et al., 1986), but absent from typical aCSF preparations, we sought to determine whether availability of glutamine limits propagation of EFPs. Addition of physiological levels of glutamine $(0.5 \mathrm{mM})$ to the aCSF for periods of $>15 \mathrm{~min}$ did not significantly alter the peak amplitude or duration of EFPs in dis- 

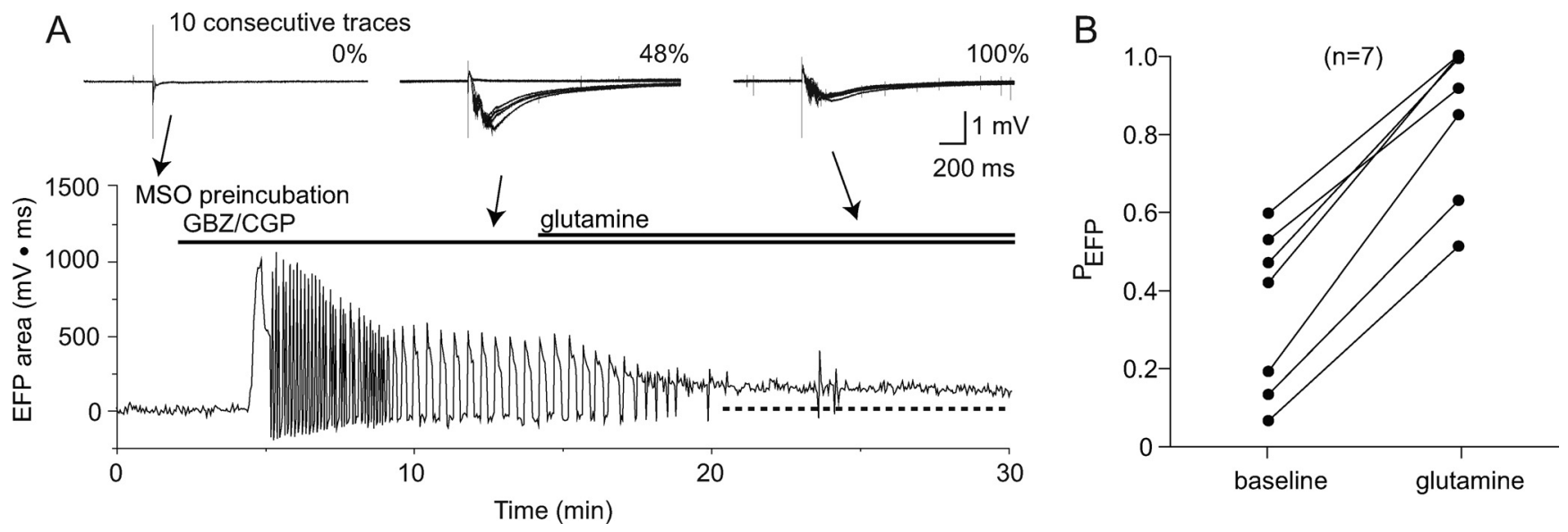

Figure 4. Partial EFP failure associated with the glutamine synthetase inhibition is reversed by exogenous glutamine. $\boldsymbol{A}$, Sample tracings (top) and time course of evoked field potential area (bottom) demonstrate, that after addition of GBZ/CGP to a neocortical slice preincubated with the glutamine synthetase inhibitor MSO, intermittent epileptiform field potentials are elicited at an ISI of $5 \mathrm{~s}$ with approximately half of the stimuli leading to polyphasic activity. Within $\sim 6$ min after the subsequent addition of $500 \mu \mathrm{m}$ glutamine to the perfusate, every stimulus leads to an epileptiform field potential. The percentage of stimuli leading to epileptiform field potentials for the different conditions is noted next to sample tracings. $\boldsymbol{B}$, Paired analysis of the probability of evoking an epileptiform field potential $\left(P_{\text {EFP }}\right)$ at baseline ( 10 min after addition of GBZ/CGP) and 15 min after the addition of $500 \mu \mathrm{m}$ glutamine indicates the consistency of this finding $(n=7 ; p<0.0001)$. Slices were preincubated with $5 \mathrm{~mm}$ MSO for $20-60$ min before recording.

inhibited slices, while addition of 5 mm glutamine routinely led to spreading depression-like events (data not shown).

A potential explanation for a lack of effect with the addition of physiological concentrations of glutamine is that endogenous glutamine is already saturating neuronal glutamine transporters, thus limiting the effect of additional glutamine. It has been suggested that the system A transporters SNAT1 and SNAT2 are the primary mediators of neuronal glutamine uptake (Chaudhry et al., 2002), and we have previously shown that in the neocortical undercut model of posttraumatic epilepsy that methylaminoisobutyric acid (MeAIB), a nonmetabolized amino acid that specifically inhibits the system A neutral amino acid transporters (Christensen et al., 1965; Mackenzie and Erickson, 2004), blocks generation of epileptiform fields (Tani et al., 2007). In addition, glutamine-induced recurrent epileptiform discharges in hippocampal slices from pilocarpine-treated chronic epileptic rats are blocked by MeAIB (Sandow et al., 2009). We therefore tested the effect of MeAIB on the generation of EFPs in the acutely disinhibited slices. Addition of up to $10 \mathrm{~mm}$ MeAIB had no effect, while $20 \mathrm{~mm}$ MeAIB induced a slight reduction in field duration without substantially affecting the peak amplitude at a variety of ISIs (Fig. $3 A, B$ ). At $5 \mathrm{~s}$ ISI, there was a small but consistent $(23.3 \pm 4 \%)$ reduction in EFP area (Fig. $3 C$ ). However in contrast to results with slices from injured neocortex (Tani et al., 2007) the probability of evoking EFPs remained at $100 \%$. These results suggest that system A transporters modulate epileptiform activity to some extent, but are not necessary to maintain prolonged excitatory network activity in pharmacologically disinhibited neocortical slices. Further, the greater effect of MeAIB on epileptiform activity previously reported with the models of chronic hyperexcitability may reflect compensatory changes, including the upregulation of the system A transporters SNAT1 and SNAT2 (Tani et al., 2007).

\section{Glutamine synthetase activity is necessary for persistent epileptiform activity}

Since glutamine is thought to be a primary precursor of synaptically released glutamate our findings were unexpected. We decided to use another approach to assess the role of glutamine in the maintenance of epileptiform activity in the acutely disinhib- ited slice. The majority of endogenous glutamine is thought to be produced by glutamine synthetase (GS), an enzyme that catalyzes the condensation of glutamate and ammonia to produce glutamine and is expressed at levels $>40$-fold higher in astrocytes than in neurons (Martinez-Hernandez et al., 1977; Albrecht et al., 2007; Cahoy et al., 2008). To determine whether glutamine derived from GS is necessary for maintaining the epileptiform fields, we examined the effects of MSO, an irreversible inhibitor of GS (Lamar and Sellinger, 1965; Manning et al., 1969). Shortly after addition of GBZ/CGP to slices preincubated with MSO (5 $\mathrm{mm}$ for $1 \mathrm{~h}$ ), EFPs were elicited by electrical stimulus, as was the case for slices not treated with MSO (Fig. 4A). Subsequent stimuli, however, led to incrementally smaller fields, and then a series of repeated failures followed by a number of evoked EFPs. This on-off pattern repeated and stabilized within $\sim 10 \mathrm{~min}$ (Fig. $4 \mathrm{~A}$ ) and without further intervention did not change over time (supplemental Fig. 2, available at www.jneurosci.org as supplemental material). To confirm that failures were a result of lack of glutamine synthesis, we added $0.5 \mathrm{~mm}$ glutamine to MSO-treated slices after a stable failure pattern developed. As a measure of response, we determined the probability that stimuli would elicit an EFP $\left(P_{\mathrm{EFP}}\right)$. The addition of glutamine led to an increase in $P_{\text {EFP }}$ in all slices tested after 15 min (Fig. $4 B$ ). In some slices the full effect of glutamine was seen as early as 5 min after adding glutamine. The mean time to full recovery (with $100 \%$ of stimuli leading to EFPs, i.e., $\left.P_{\mathrm{EFP}}=1\right)$ was $12.8 \pm 9.7 \mathrm{~min}(n=7)$. The robust response to glutamine in the MSO-treated slices is consistent with inhibition of astrocytic glutamine synthetase as the primary effect of MSO.

\section{Non-system A transport system contributes to neuronal glutamine uptake}

The effect of MSO in inducing failures of epileptiform events was dramatic and indicated that astrocytic glutamine synthesis is necessary for maintenance of polyphasic network activity in disinhibited cortical slices. It follows that glutamine transfer to neurons should also be required for sustained epileptiform fields. However, in our experiments MeAIB at $20 \mathrm{~mm}$ [a concentration that completely inhibits system A transport (Mackenzie and Erickson, 2004)] had only a subtle effect on the size of EFPs and 
A
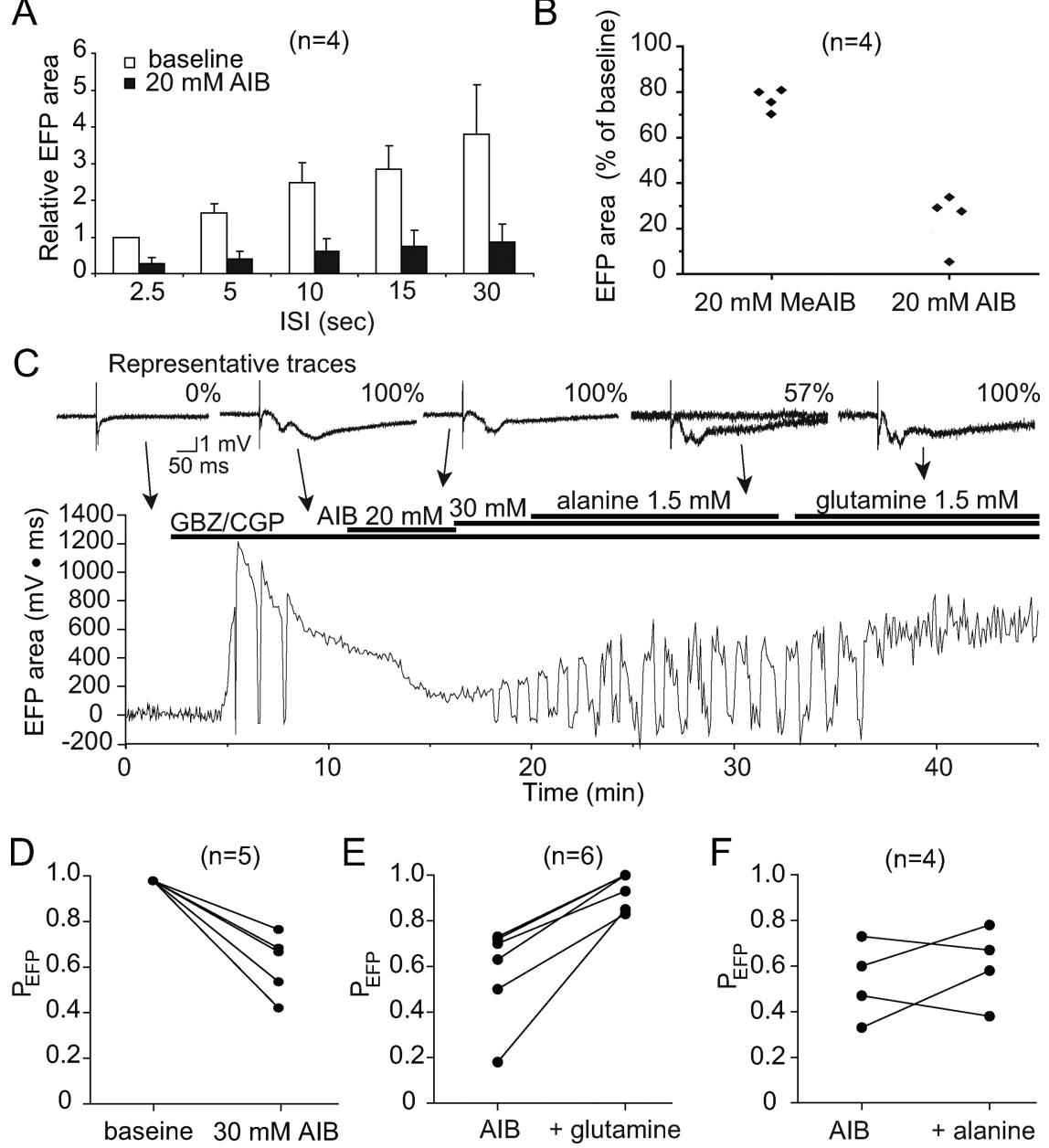

B
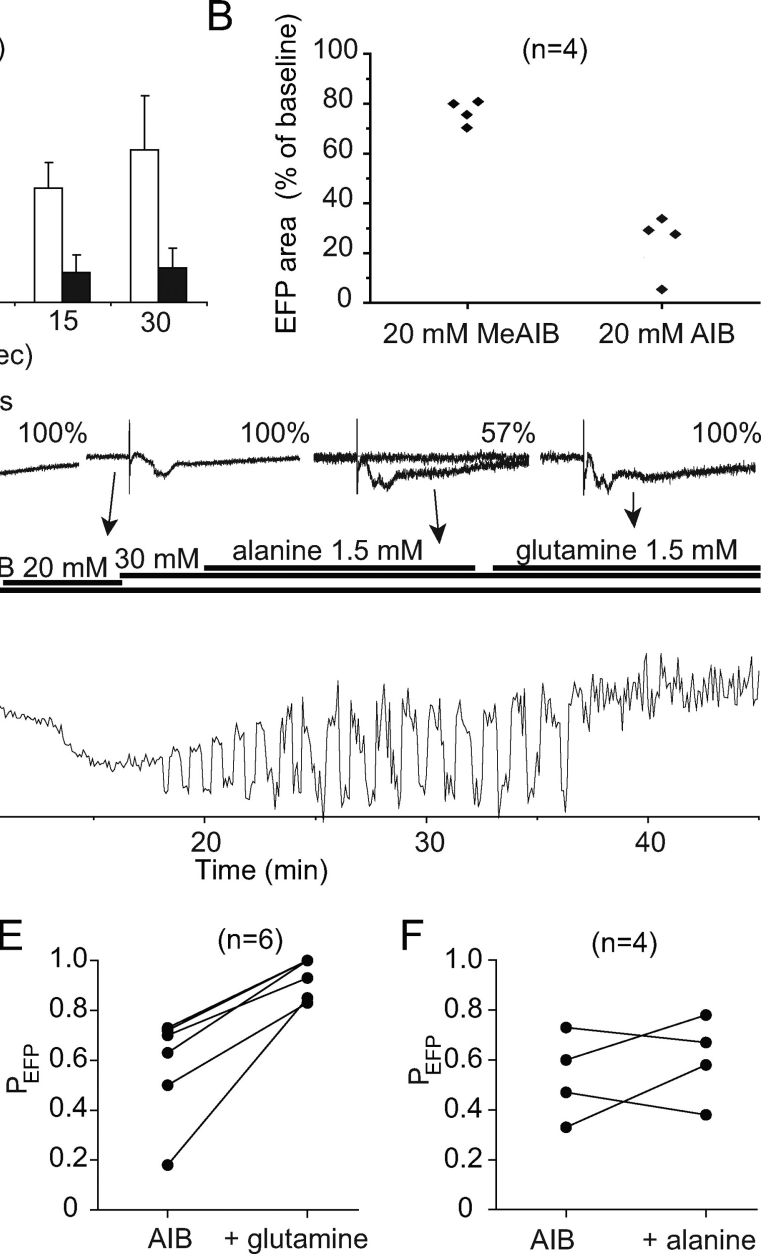

$\mathrm{F}$

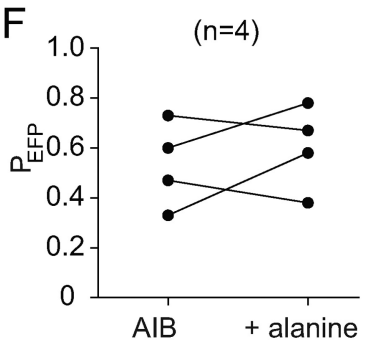

Figure 5. The neutral amino acid analog AIB decreases the probability of evoking epileptiform field potentials. $A$, Comparison of area of evoked field potentials of GBZ/CGP-treated slices at 2.5-30 $\mathrm{s}$ ISI in the absence of (baseline, open bars) and 10 min after the addition of $20 \mathrm{~mm}$ AIB (black bars) indicates a marked reduction with AIB treatment at all ISIs $(n=4)$. B, Scatter plots of EFP areas (as a percentage of the baseline EFP area in slices stimulated at $5 \mathrm{~s} \mid \mathrm{SI}$ ) indicate that the residual fields potentials are significantly smaller in the presence of AIB than MeAIB ( $24 \pm 13 \%$ and $77 \pm 5 \%$, respectively; $n=4$ in each group; $p<0.0003$ ). EFPs were measured $10 \mathrm{~min}$ after addition of $20 \mathrm{~mm}$ MeAIB or AIB to the perfusate. C, Example traces of evoked field potentials (top) and time course of evoked field potential area (bottom) from a single cortical slice demonstrate the sequential development of large field potentials with GBZ/GGP; a reduction in size of the fields with $20 \mathrm{~mm}$ AIB; followed by intermittent fields with $30 \mathrm{~mm}$ AIB; and restoration of the responses ( $100 \%$ successes) with glutamine but not isotonic alanine. The percentage of stimuli leading to epileptiform field potentials for the different conditions is noted next to the sample traces. $D$, Paired analysis of $P_{\text {EFP }}$ in GBZ/CGPtreated slices at baseline and 5 min after addition of $30 \mathrm{~mm}$ AIB demonstrates a consistent reduction in probability with AIB $(n=$ 6; $p<0.0001$ ). $E$, Paired analysis of $P_{\text {EFP }}$ demonstrates that addition of $1.5 \mathrm{~mm}$ glutamine to AlB-treated slices with intermittent epileptiform field potentials leads to a consistent increase in $P_{\text {EFP }}(n=6 ; p<0.0008) . \boldsymbol{F}$, In contrast, addition of $1.5 \mathrm{~mm}$ alanine is not associated with a significant change in $P_{\text {EFP }}(n=4 ; p=0.47)$. Evoked field potentials were analyzed 5 min after the addition of either alanine or glutamine.

did not alter the probability of epileptiform events, raising the possibility that another glutamine transporter might be involved in maintaining astrocyte to neuron glutamine transfer. We therefore tested the effect of histidine and alanine-small neutral amino acids that are substrates and competitive inhibitors for a number of glutamine transporters (Bröer and Brookes, 2001). When either of these amino acids was added to the perfusate (at $10 \mathrm{~mm}$ ), the size and frequency of the EFPs was reduced (supplemental Fig. 3, available at www.jneurosci.org as supplemental material), but spreading depression-like events (SD) invariably followed and limited further analysis.

Since SD could result from entry of these amino acids into intracellular metabolic pathways, we tested the effect of AIB, a broad spectrum inhibitor of amino acid transport (Christensen,
1962). Similar to MeAIB, AIB is a nonmetabolized neutral amino acid analog that is readily transported into mammalian cells, but AIB lacks the $\alpha$-methyl substitution that confers selective inhibition of system A transport (Christensen, 1962; Christensen et al., 1965; Appelgren et al., 1982). We found that $20 \mathrm{~mm}$ AIB significantly reduced the area of EFPs at all ISIs (Fig. $5 A$ ). A comparison of the effect of adding $20 \mathrm{~mm}$ MeAIB to that of adding $20 \mathrm{~mm}$ AIB on EFP area during stimulation with an ISI of $5 \mathrm{~s}$ indicates a far greater effect with AIB (Fig. 5B). Addition of $30 \mathrm{~mm}$ AIB induced intermittent failures similar to what was seen with MSO (Fig. $5 C, D$ ). If the effect of AIB was due to competitive inhibition of endogenous glutamine, then addition of exogenous glutamine at supraphysiological concentrations should overcome the effect of AIB. Indeed, we found an increase in the probability of evoking an EFP with the addition of $1.5 \mathrm{~mm}$ glutamine (Fig. $5 E$ ), but not $1.5 \mathrm{~mm}$ alanine (Fig. $5 F$ ).

To confirm the predicted biochemical and metabolic effects of AIB treatment, we examined amino acid levels and glutamine metabolism using mass spectrometry and radiotracer studies. After $30 \mathrm{~min}$ of electrophysiological analysis with our standard stimulation protocol, slices were removed from the recording rig and the neocortex isolated. Mass spectrometry was then used to determine the amino acid concentrations in ethanol extracts of the tissues samples. As expected, preincubation with MSO was associated with markedly decreased glutamine levels in the neocortex (Fig. 6A). AIB incubation lead to a smaller, but also significant, decrease in glutamine levels while MeAIB had no effect on glutamine (Fig. 6A). The greater effect with MSO compared to AIB is consistent with AIB blocking neuronal uptake of glutamine released from astrocytes, while not affecting the larger astrocytic pool of glutamine that is targeted by inhibition of glutamine synthetase with MSO (Fonnum, 1993). Somewhat surprisingly, we found little effect on glutamate levels with the MSO or AIB treatment (Fig. 6B). The lack of correlation between the effect on bulk glutamine and glutamate levels may reflect the fact that the neurotransmitter pool of glutamate is much smaller than the larger general metabolic pool (Erecińska and Silver, 1990).

To test whether inhibiting glutamine uptake with AIB could reduce glutamate available for synaptic release, we next examined the fate of exogenously supplied radiolabeled glutamine in slices. For these experiments, neocortical slices were incubated in oxygenated $\mathrm{Mg}^{2+}$-free ACSF without perfusion, an incubation condition that leads to frequent spontaneous epileptiform events (data not shown) and approximates the activity level of the electrically stimulated GBZ/CGP-treated slices used in our electrophysiology analyses. As expected, AIB caused a reduction of 
$\left[{ }^{3} \mathrm{H}\right]$ glutamine uptake (Fig. $6 \mathrm{C}$ ). MeAIB also reduced glutamine uptake, although to a much lesser extent. At a lower concentration $(10 \mathrm{~mm})$, a similar, but less robust, effect on glutamine uptake also occurred with AIB (supplemental Fig. 4, available at www.jneurosci.org as supplemental material). To determine whether the glutamine taken up into the slice is converted to glutamate for release, we used ionexchange chromatography to isolate glutamate from free amino acid extracts of the tissue and the incubation media. Radioactivity in the glutamate fraction of tissue extracts was reduced in the AIBtreated slices to an extent similar to the reduction in glutamine uptake (Fig. $6 D)$. Glutamate derived from $\left[{ }^{3} \mathrm{H}\right]$ glutamine and released into the incubation media in a pulse-chase protocol (Fig. $6 E$ ) was also reduced in the AIB-treated slices. MeAIB tended to reduce $\left[{ }^{3} \mathrm{H}\right]$ glutamate levels in both the tissue and incubation media, but not to a statistically significant extent. These findings indicate that AIB inhibits neuronal uptake of glutamine that contributes to the synthesis of glutamate that is subsequently released.

\section{De novo synthesis is necessary for the maintenance of epileptiform activity} The results of MSO and AIB inhibition suggest that transfer of astrocyte-derived glutamine to neurons is necessary to maintain polyphasic network activity. Although synaptically released glutamate taken up by astrocytes provides some of the GS substrate, astrocytes are also capable of de novo synthesis of glutamate (Kvamme, 1998; McKenna, 2007). A primary pathway for this is the amino transferase catalyzed amination of $\alpha$-ketoglutarate, an intermediate in the TCA cycle. Because astrocytes express pyruvate carboxylase, they are capable of anaplerosis (McKenna, 2007), the net synthesis of TCA intermediates. As a result astrocytes can generate $\alpha$-ketoglutarate (and therefore glutamate) from glucose. Neurons, however, lack appreciable levels of pyruvate carboxylase (Shank et al., 1985; McKenna, 2007) and have a limited capacity for de novo glutamate synthesis. The de novo anaplerotic pathway for glutamate synthesis in astrocytes may contribute up to $50 \%$ to the pool of synaptically released glutamate in vivo (Oz et al., 2004). In the perfused slice, there may be greater reliance on this pathway as some synaptically released glutamate is washed out (Kapetanovic et al., 1993). It has been noted that the extracellular space of hippocampal tissue fixed after slicing is greater than that of perfusion-fixed hippocampal tissue (Furness et al., 2008). This suggests that an increase in extracellular volume could contribute to the inefficiency of the glutamate-glutamine cycle and a greater dependence on anaplerosis for releasable glutamate in slices. However, diffusion measurements of the extracellular volume in $400 \mu \mathrm{m}$ slices indicate that the relative extracellular space is similar to that for intact brain (Hrabetová and Nicholson, 2000; Syková and Nicholson, 2008). To determine whether de novo synthesis con- tributes to glutamate release, we next tested the effect of AOAA, a broad spectrum inhibitor of amino transferases (Geng et al., 1997), on EFPs. Cortical slices were either preincubated or perfused with 1-5 mM AOAA for $\sim 1 \mathrm{~h}$. Following AOAA treatment and the further addition of GBZ/CGP, no EFPs were obtained with a typical $(150 \mu \mathrm{A})$ stimulation. Increasing stimulus current led to the induction of large EFPs that diminished progressively until failures were elicited (Fig. 7A). Further increasing the stimulus intensity led to the induction of large EFPs, but again failures ensued soon after. In contrast to the effects of MSO or AIB, AOAA eventually led to an almost complete lack of evoked EFPs. Addition of $500 \mu \mathrm{M}$ alanine did not increase $P_{\mathrm{EFP}}$ (Fig. 7B), but $500 \mu \mathrm{M}$ glutamine consistently increased $P_{\mathrm{EFP}}$ within $15 \mathrm{~min}$ (Fig. $7 C)$. Full recovery was noted in all slices treated with glutamine $(11.2 \pm 4.1 \mathrm{~min}$ to recovery, $n=11)$. These results suggest that $d e$ novo glutamate synthesis through the anaplerotic pathway is necessary for maintaining excitatory network in the slice when glutamine is absent from the perfusate.

Entry of exogenous glutamate into the neurotransmitter pool requires a glutamine intermediate

The effect of MSO and AIB on EFPs and the reversibility of the effect with glutamine suggest that neuronal glutamine uptake is necessary to maintain hyperexcitability. The observation that EFPs were completely depleted in AOAA further suggests that 
A 10 consecutive traces
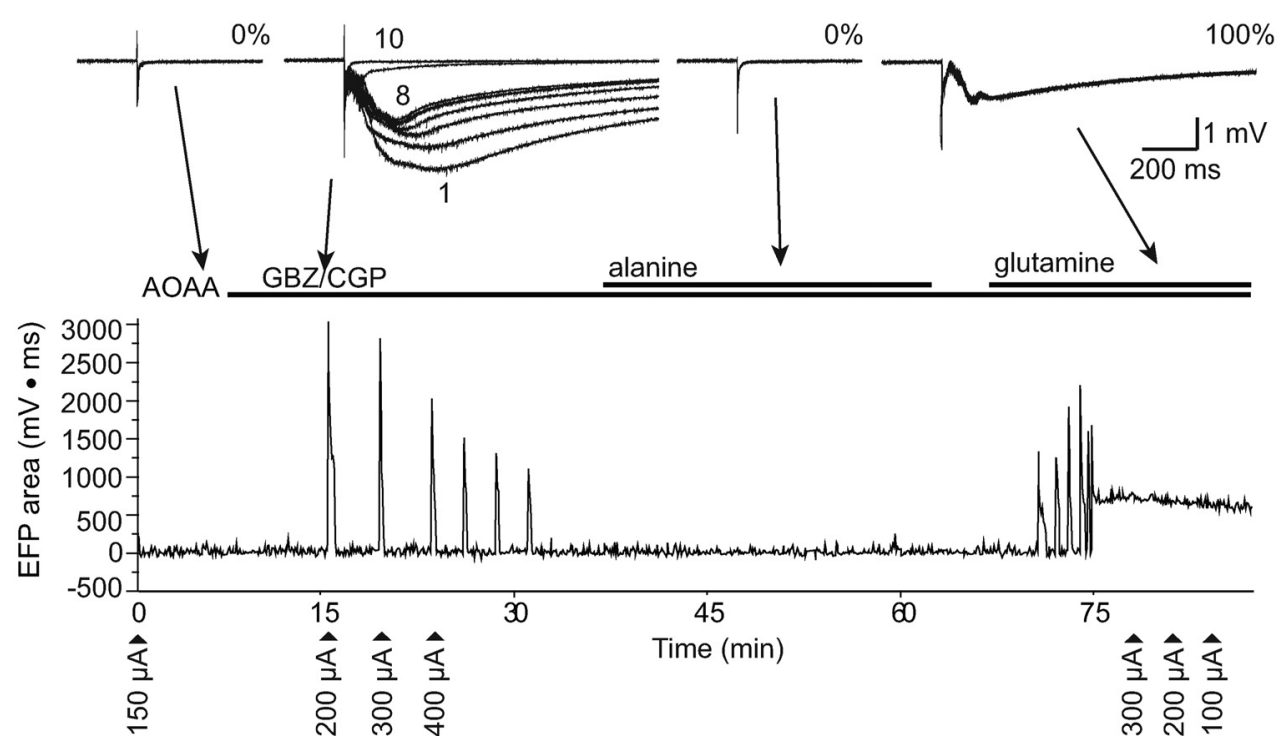

B
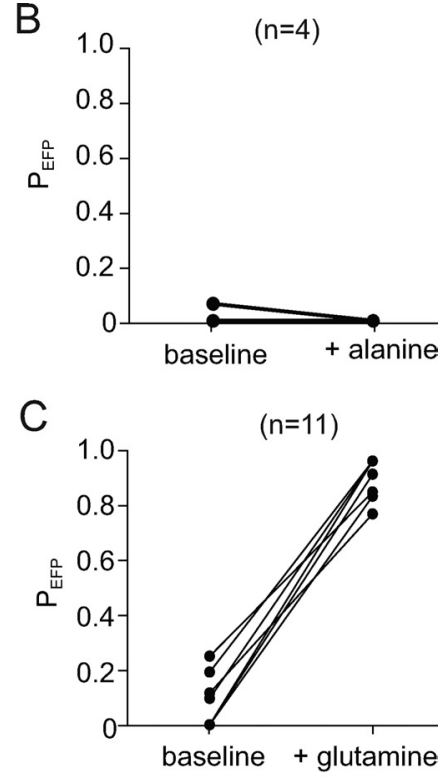

Figure 7. The amino transferase inhibitor AOAA markedly reduces probability of evoking EFPs. A, Sample traces (top) and time course of evoked field potential area (bottom) from field recordings of a cortical slice continuously treated with AOAA for $1 \mathrm{~h}$ before the recording and throughout the recording period. No EFPs were obtained with a typical (150 $\mu \mathrm{A})$ stimulation, even after wash-in of GBZ/CGP. Increasing stimulus current (indicated by arrowheads) led to transient generation of EFPs with a large initial field potential followed by successively smaller field potentials and ultimately failures [second set of sample tracings at top; 10 consecutive sweeps with position in order of sweeps indicated by numbers - the first sweep (1) was the first epileptiform field potential, and the last (10) was a failure]. Subsequent addition of $500 \mu \mathrm{m}$ alanine had no effect, but addition of $500 \mu \mathrm{m}$ glutamine led to consistent epileptiform field potentials with each stimulus. $\boldsymbol{B}$, Paired analysis demonstrates no significant effect of alanine on $P_{\text {EFP }}$ in A0AA-treated slices $(n=4 ; p=0.39)$. C, Glutamine $(500 \mu \mathrm{M})$ produced a robust increase in $P_{\text {EFP }}$ in all slices $(n=11 ; p<0.00001)$.

glutamate recycling is inefficient. Although the glutamine rescue of EFPs in the AOAA-treated slices confirms that neuronal glutamine uptake can maintain epileptiform activity in the absence of de novo glutamate synthesis, it is unclear whether the entire glutamate-glutamine cycle is functionally intact in the slice. If glutamate that is removed from the synapse by astrocytes can be recycled and released, then addition of exogenous glutamate should rescue EFPs in AOAA-treated slices. Conversely, since glutamate is primarily taken up by astrocytes (Danbolt, 2001) and a glutamine intermediate is required for transfer to neurons (Chaudhry et al., 2002), glutamate should not rescue MSOtreated slices. To test whether exogenous glutamate enters the glutamate-glutamine cycle, we applied $250 \mu \mathrm{M}$ glutamate to the perfusate of AOAA-treated slices. The addition of glutamate to the perfusate increased $P_{\mathrm{EFP}}$ to $100 \%$ in all slices $(21.8 \pm 7.6 \mathrm{~min}$, $n=10$ ) (Fig. $8 A, B$ ). In contrast, with MSO-treated slices, $P_{\mathrm{EFP}}$ remained unchanged when glutamate was added (Fig. $8 C, D$ ). In some cases, addition of glutamate to MSO-treated slices was associated with a small reduction in the size of evoked fields during the "on" periods, suggesting the possibility of glutamate receptor desensitization or activation of other mechanisms that downregulate glutamate signaling. However, the invariable appearance of EFPs in the AOAA-treated slices after addition of glutamate indicates that any such effect does not preclude the generation of evoked epileptiform activity. Further, we have previously demonstrated that glutamate is efficiently taken up in the neocortical slice by TBOA-sensitive transporters and that adding glutamate at this concentration has little effect on bulk steady-state extracellular levels (Dulla et al., 2008). The lack of an effect on $P_{\text {ЕFP }}$ with addition of glutamate to MSO-treated slices suggests that neuronal presynaptic glutamate uptake, if present, is not sufficient to maintain high levels of synaptic glutamate release required for EFPs and that conversion to glutamine in astrocytes is required. It follows that the incorporation of exogenously added glutamate to the neurotransmitter pool in the AOAA-treated slices requires neuronal uptake of astrocyte-derived glutamine. Further supporting this, blocking glutamine transport with addition of the broad spectrum glutamine uptake antagonist histidine $(10 \mathrm{~mm})$ inhibited the glutamate rescue of EFPs in AOAA-treated slices (supplemental Fig. 5A, $B$, available at www.jneurosci.org as supplemental material). In these experiments addition of histidine did not lead to SD, suggesting that the activity of the amino transferases inhibited by AOAA is necessary for inducing SD with histidine.

Attenuation of epileptiform fields by AIB is associated with a reduction in frequency and amplitude of spontaneous EPSCs To begin to address whether inhibition of neuronal glutamine uptake is associated with a reduction in glutamate release at a synaptic level, we recorded from layer $\mathrm{V}$ pyramidal neurons in disinhibited slices. Whole-cell voltage-clamp recordings in the presence of GBZ/CGP exhibited a pattern of intracellular currents similar to the extracellular field recordings, with both short latency and late, prolonged polysynaptic components (Fig. 9A, top traces). The amplitude of whole-cell voltage-clamp responses was plotted as area (charge of EFP associated synaptic current responses) versus time as previously done with EFPs (Fig. 9A, bottom). To disrupt glutamate-glutamine cycle, we chose AIB because its effect was readily reversible. When AIB was added to GBZ/CGP-treated slices, the large evoked intracellular currents began to exhibit the intermittent (on-off) pattern seen with extracellular field recordings under the same conditions, showing that the synaptic inputs onto layer $V$ neurons reflect the networks that are monitored with the field recordings. The early fixed latency response occurring within $8 \mathrm{~ms}$ after the stimulus artifact, which likely reflects the monosynaptic inputs onto the patched 
cell, was markedly reduced with addition of AIB (Fig. 9B, top and middle tracings). This resolved with washout (Fig. $9 B$, bottom tracing). AIB reduces the peak amplitude to $52.90 \pm 7.28 \%$ of the GBZ/CGP baseline (Fig. 9C) $(n=8 ; p<0.00001)$ and with washout the peak amplitude increased to a level not statistically different from the GBZ/CGP baseline (89.82 \pm $27.86 \% ; n=8 ; p=0.37)$.

To estimate the influence of disrupting glutamate-glutamine cycle on excitatory transmission at the synaptic level, we also examined changes in frequency and amplitude of spontaneous events in the intervals between evoked responses. Typical tracings from a GBZ/CGPtreated slice show a marked decrease in the frequency of spontaneous EPSCs with addition of AIB (Fig. 9D). In cells from 10 slices with addition of AIB, there was a decrease in spontaneous event frequency to approximately onefourth that in the presence of GBZ/CGP alone (Fig. 9E). This effect was partially reversible upon washout of AIB. Addition of AIB was also associated with a reduction in the size of the spontaneous events as noted by a leftward shift in the cumulative probability versus amplitude plot (Fig. 9F) and paired analysis of the mean amplitude spontaneous events (Fig. 9G) $(p<0.01, n=10$ paired $t$ test). In both cases, the decrease in amplitude was $\sim 1 \mathrm{pA}$.

\section{Discussion}

Although it is a well established metabolic pathway in the vertebrate brain, the necessity of the glutamate-glutamine cycle for excitatory neurotransmission remains controversial (Rothman et al., 2003; Kam and Nicoll, 2007). In this study, we provide evidence that a fundamental part of the cycle, the transfer of astrocyte-derived glutamine to neurons, is essential for sustained epileptiform activity in acutely disinhibited neocortical slices. We demonstrate that the cycle contributes to excitatory neurotransmission in our slices and that exogenously supplied glutamate can enter the cycle. However, we find that in addition to the cycle, de novo synthesis, which is occurring predominantly in astrocytes, is required for maintaining high levels of glutamate release in our in vitro system. We also find, in contrast to previous suggestions, that the cycle can function independently of system A transporters and uses an, as of yet, unidentified neuronal glutamine transporter or transporters. Finally we find that the attenuation of network activity through inhibition of glutamine transport is associated with reduced frequency and amplitude of spontaneous events detected at the single-cell level, suggesting that availability of glutamine influences neurotransmitter release at excitatory synapses during periods of intense network activity.

Although the majority of synaptically released glutamate is derived from the glutamate-glutamine cycle both in vivo and in vitro (Lieth et al., 2001; Sibson et al., 2001), glutamate can be derived from a number of other precursors which could explain the limited effect of disrupting the cycle pharmacologically or genetically on behavior and synaptic transmission (Blin et al., 2002; Masson et al., 2006; Kam and Nicoll, 2007). While likely crucial for periods of increased neuronal activity ( $\mathrm{Oz}$ et al., 2004), these other pathways complicate the characterization of the glutamate-glutamine cycle, particularly in ex vivo preparations. The acutely disinhibited rat neocortical slice provides us with a system in which the influence of the glutamateglutamine cycle on excitatory synaptic transmission can be assessed in a physiologically intact system, and under conditions in which potentially confounding effects on metabolism of the inhibitory neurotransmitter GABA are rendered irrelevant.

Our finding of a marked reduction in epileptiform activity when slices are treated with MSO coupled with the minimal effect of MeAIB indicates that system A transport is not necessary for neuronal uptake of glutamine. This is consistent with previous studies that have suggested that the system A transporters SNAT1 and SNAT2 are unlikely to play a crucial role in the glutamateglutamine cycle and evidence for system A-independent neuronal glutamine transport (Rae et al., 2003; Conti and Melone, 2006). However, a recent study suggests that MeAIB-sensitive transport is a major pathway for neuronal glutamine uptake in 
A Average of 10 traces

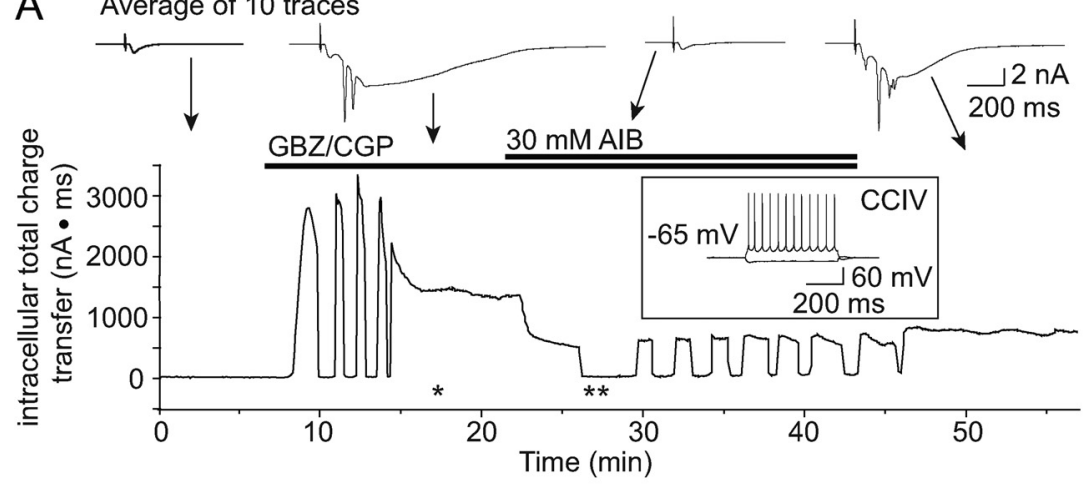

B

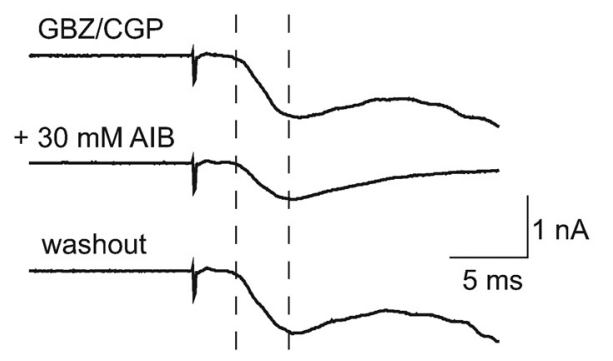

D

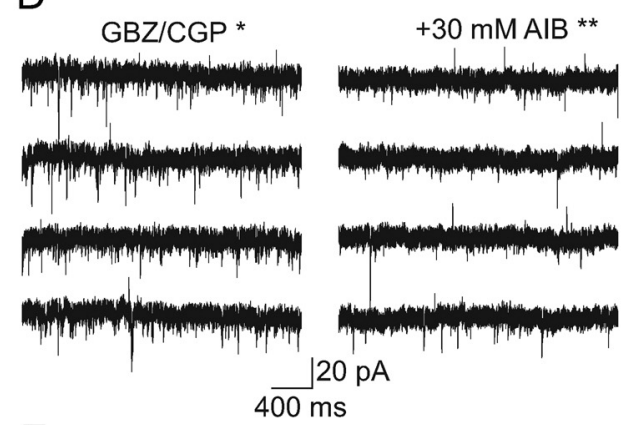

$\mathrm{F}$

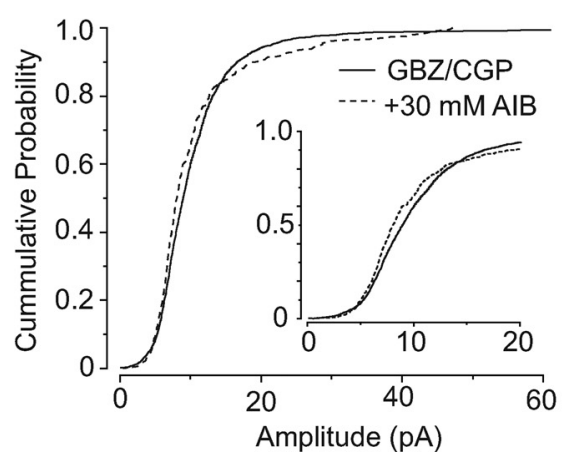

C 총응

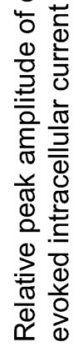

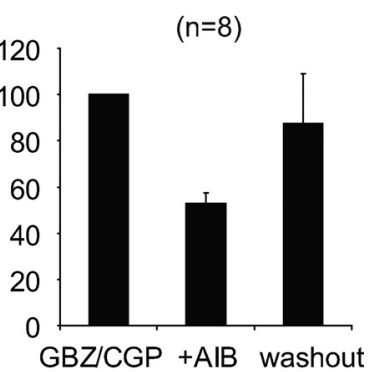

$E$

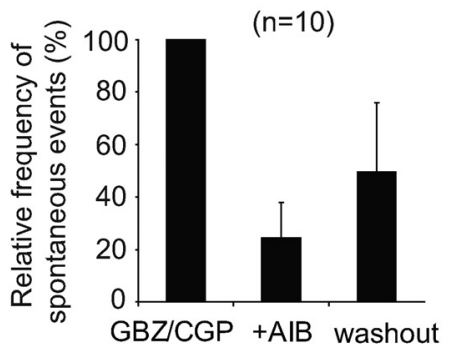

G

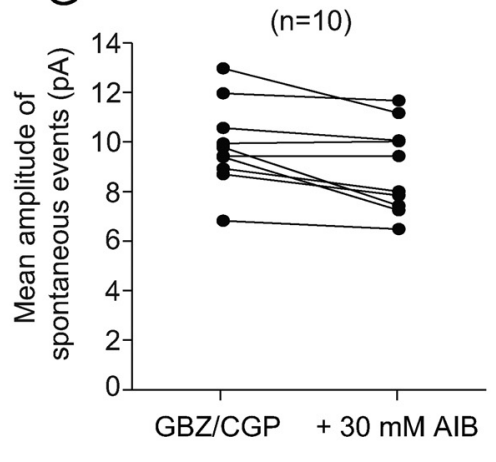

Figure 9. Frequency and amplitude of spontaneous events are reduced with addition of AIB to GBZ CGP-treated neocortical slices. A, Sample traces (top) and time course (bottom) of charge transfer detected through intracellular voltage-clamp recording of a layer $V$ pyramidal neuron demonstrate findings that parallel field recordings. Note increased response duration with GBZ/CPG wash-in and reduction following addition of AIB. Also note that after washout of AIB each stimulus is associated with a prolonged polyphasic current ( $100 \%$ successes), while with addition of AIB intermittent polyphasic currents ( $\sim 30-40 \%$ successes) were noted. Bars indicate times of drug perfusion. Inset shows voltage responses to hyperpolarizing and depolarizing intracellular current injections. This cell and all others in this set of intracellular experiments demonstrated typical features of layer V pyramidal neurons, including adapting spike rates and hyperpolarization-induced membrane potential sag. $\boldsymbol{B}$, Sample tracings of short latency responses demonstrate a reduction in the current with addition of AIB (middle tracing) compared to GBZ/CGP alone (top tracing) and after washout (bottom tracing). Dashed lines indicate fixed latency of onset (left line) and peak (right line) of postsynaptic current, consistent with monosynaptic events. $C$, Comparison of peak amplitudes of the short latency responses after addition of $30 \mathrm{~mm}$ AIB indicates a reduction to $52.90 \pm 7.28 \%$ of the responses in GBZ/CGP alone ( $n=8, p<0.0001)$. With

vivo (Kanamori and Ross, 2006). In addition, we and others have demonstrated that in models of chronic hyperexcitability system A transport is required for sustained epileptiform activity (Tani et al., 2007; Sandow et al., 2009), suggesting that with chronic hyperexcitability, system A transporters contribute more significantly to the glutamate-glutamine cycle. The demonstration that MeAIB attenuates epileptiform activity in acutely disinhibited hippocampal slices (Bacci et al., 2002), also suggests that the role of system A transport in the glutamate-glutamine cycle varies from brain region to brain region.

The molecular identity of the MeAIBinsensitive transporter or transporters is unknown. Unfortunately, AIB, alanine, and histidine, which all appear to disrupt the cycle, broadly inhibit glutamine transporters (Christensen, 1962; Bröer and Brookes, 2001). MeAIB-insensitive neuronal glutamine transport in neurons and synaptosomes has been described ( $\mathrm{Su}$ et al., 1997; Tamarappoo et al., 1997; Rae et al., 2003), but further biochemical and molecular studies will be needed to more completely characterize this step in the glutamate-glutamine cycle.

The rapid run down of evoked epileptiform fields in AOAA-treated slices indicates that recycling alone cannot maintain the level of glutamate release required for epileptiform field generation in our system. The rescue of the epileptiform fields in these slices with glutamate does, however, confirm that the glutamate-glu-

$\longleftarrow$

washout the peak amplitude was similar to that of the GBZ/ CGP baseline $(89.82 \pm 27.86 \% ; n=8 ; p=0.37)$. $D$, Current recordings from a single cell collected from the $3 \mathrm{~s}$ before the next stimulus during a $0.2 \mathrm{~Hz}$ stimulation paradigm demonstrate spontaneous activity after addition of GBZ CGP (left) and less frequent and smaller events in the same cell with subsequent addition of AIB to the perfusate (right). Recordings are from the same cell depicted in $\boldsymbol{A}$, and asterisk and double asterisk indicate time points at which these tracings were obtained. $\boldsymbol{E}$, Plot of the frequency of spontaneous events normalized to GBZ/CGP conditions demonstrates a marked and reversible reduction by $30 \mathrm{~mm}$ AIB (AIB: $24.25 \pm 13.39 \%$ of control and washout: $49.50 \pm 26.52 \%$ of control; $n=10$ $p<0.0001$ and $p<0.03$, respectively). $\boldsymbol{F}$, Cumulative histogram of spontaneous events recorded from the cell depicted in $\boldsymbol{A}$ shows a reduction in the median amplitude of events after addition of AIB. G, Paired analysis from single-cell recordings demonstrates a consistent decrease in the mean amplitude of the spontaneous events with the addition of AIB (GBZ/CGP, $9.89 \mathrm{pA}$; $+30 \mathrm{~mm}$ AlB, $8.97 \mathrm{pA}$; difference, $0.92 \mathrm{pA} ; n=10$; $p<0.05)$. To maintain consistent solution osmolarity throughout the intracellular recordings, a modified aCSF with reduced $\mathrm{NaCl}(111 \mathrm{~mm})$, and either $30 \mathrm{~mm}$ mannitol or $30 \mathrm{~mm}$ AlB was used. 
tamine cycle is intact in our slices. The far greater expression level of pyruvate carboxylase, an enzyme required for anaplerosis, in astrocytes compared to neurons suggests that most de novo synthesis occurs in astrocytes and that the glutamate is converted to glutamine and then transferred to neurons (Shank et al., 1985). However, if glutamine synthetase and the neuronal glutamine transport systems are completely inhibited by MSO and AIB, then the more complete response to AOAA (e.g., the absence of intermittent epileptiform events) suggests in addition that some de novo synthesis occurs in neurons where glutamate can be packaged directly into vesicles without going through a glutamine intermediate. This interpretation would be consistent with the capacity of cultured neurons to sustain glutamate release in the absence exogenous glutamine (Kam and Nicoll, 2007).

Our field recording data suggest that disruption of the glutamateglutamine cycle attenuates glutamatergic neurotransmission. This presumably results from a limited availability of glutamate. With less glutamate available for vesicular packaging, it follows that the amount in each vesicle (e.g., quantal size) will be reduced, and in the extreme case, vesicles will contain no detectable glutamate (Wolosker et al., 1996). This would be tested most directly by measuring miniature EPSPs in the presence of tetrodotoxin (Katz and Miledi, 1965). However, the requirement for frequent activation of neuronal networks in our system precludes the use of tetrodotoxin. Our alternative approach, analyzing spontaneous synaptic events with whole-cell voltage-clamp recordings, revealed a reduction in amplitude and a far greater decrease in frequency of events. The reduction in amplitude of spontaneous events is consistent with a decrease in miniature excitatory postsynaptic events and therefore quantal size (Min and Appenteng, 1996). However, the small reduction $(\sim 10 \%)$ would not explain the more marked effect on epileptiform events or even the monosynaptic early evoked responses recorded from the same cells.

The reduction in spontaneous EPSC frequency $(\sim 75 \%)$ with AIB is more consistent with the effect on epileptiform events and the reduction in the early evoked responses. The simplest explanation for the reduction in frequency of spontaneous events is a decreased probability of release (Katz, 1962). Unfortunately, the need for consistent intermittent stimulation in our system precludes the use of standard measures of release probability and vesicle fusion such as paired-pulse ratio and FM1-43 destaining kinetics (Katz and Miledi, 1968; Pyle et al., 1999). Further, it is not directly obvious how AIB would cause a reduction in probability of vesicle fusion. An alternative explanation for the reduced frequency of spontaneous events is that some terminals that form synapses onto the recorded cell are recruited into the activated network and are readily depleted of glutamate when neuronal glutamine transport is blocked. Vesicles from these terminals would continue to fuse with the same probability, but no detectable glutamate would be released. The spontaneous events and persisting monosynaptic evoked responses that are detected in the presence of AIB could arise from terminals that are perhaps less activated by the recurrent activity of the epileptiform event.

Can disrupting the glutamate-glutamine cycle be used to treat epilepsy? Our data and that of others suggest that it can (Bacci et al., 2002; Tani et al., 2007; Eid et al., 2008). However, recent work has demonstrated that cycle activity is impaired in epileptic tissue (Pan et al., 2008). These contradictory findings may be explained, in part, by the system being studied and which step in the cycle is disrupted. Blocking the cycle after the synaptic release of glutamate but before synthesis of glutamine would lead to a paradoxical increase in excitability as synaptic glutamate will not be efficiently cleared from the extracellular space (Rothstein et al., 1996; Tanaka et al., 1997). Indeed inhibiting astrocytic glutamate transporters increases epileptiform activity (Dulla et al., 2008). In addition, MSO leads to seizures when given to rodents (Rowe and Meister, 1970), and cases of human temporal lobe epilepsy have been associated with a reduction in astrocytic expression of glutamine synthetase (Eid et al., 2004). The absence of a similar effect in our slices treated with MSO may reflect the fact that synaptic glutamate does not readily build up in perfused brain slices. Disrupting the cycle after glutamine synthesis, but before glutamate release (either at the intercellular transfer of glutamine, synthesis of glutamate, or vesicular packaging and release), should lead to a decrease in synaptic levels of the neurotransmitter. Inhibiting neuronal glutamine uptake is particularly appealing as the primary metabolic effect would be an increase in extracellular glutamine. While blocking neuronal glutamine uptake might also inhibit synthesis of GABA (Liang et al., 2006; Fricke et al., 2007), an undesirable effect when trying to treat hyperexcitability, our data suggest that MeAIB-sensitive transporters, which appear to be important in GABA synthesis, are not essential to the glutamate-glutamine cycle. Thus identifying and targeting the MeAIB-insensitive neuronal glutamine transporters in the cycle may be of particular importance.

\section{References}

Albrecht J, Sonnewald U, Waagepetersen HS, Schousboe A (2007) Glutamine in the central nervous system: function and dysfunction. Front Biosci 12:332-343.

Appelgren LE, Lindqvist I, Nilsson O, Ronquist G (1982) Distribution of some non-physiological amino acids (AIB, DAB, MeAIB) in mice. Acta Physiol Scand 115:261-268.

Aram JA, Lodge D (1988) Validation of a neocortical slice preparation for the study of epileptiform activity. J Neurosci Methods 23:211-224.

Bacci A, Sancini G, Verderio C, Armano S, Pravettoni E, Fesce R, Franceschetti S, Matteoli M (2002) Block of glutamate-glutamine cycle between astrocytes and neurons inhibits epileptiform activity in hippocampus. J Neurophysiol 88:2302-2310.

Blin M, Crusio WE, Hévor T, Cloix JF (2002) Chronic inhibition of glutamine synthetase is not associated with impairment of learning and memory in mice. Brain Res Bull 57:11-15.

Bröer S, Brookes N (2001) Transfer of glutamine between astrocytes and neurons. J Neurochem 77:705-719.

Broman J, Hassel B, Rinvik E, Ottersen OP (2000) Biochemistry and anatomy of transmitter glutamate. In: Glutamate (Ottersen OP, StormMathisen J, eds), pp 1-44. Amsterdam: Elsevier.

Brugger F, Wicki U, Olpe HR, Froestl W, Mickel S (1993) The action of new potent $\mathrm{GABAB}$ receptor antagonists in the hemisected spinal cord preparation of the rat. Eur J Pharmacol 235:153-155.

Cahoy JD, Emery B, Kaushal A, Foo LC, Zamanian JL, Christopherson KS, Xing Y, Lubischer JL, Krieg PA, Krupenko SA, Thompson WJ, Barres BA (2008) A transcriptome database for astrocytes, neurons, and oligodendrocytes: a new resource for understanding brain development and function. J Neurosci 28:264-278.

Chaudhry FA, Reimer RJ, Edwards RH (2002) The glutamine commute: take the N line and transfer to the A. J Cell Biol 157:349-355.

Christensen HN (1962) Biological transport. New York: W. A. Benjamin.

Christensen HN, Oxender DL, Liang M, Vatz KA (1965) The use of $\mathrm{N}$-methylation to direct route of mediated transport of amino acids. J Biol Chem 240:3609-3616.

Conti F, Melone M (2006) The glutamine commute: lost in the tube? Neurochem Int 48:459-464.

Courtney KR, Prince DA (1977) Epileptogenesis in neocortical slices. Brain Res 127:191-196.

Danbolt NC (2001) Glutamate uptake. Prog Neurobiol 65:1-105.

Darmaun D, Manary MJ, Matthews DE (1985) A method for measuring 
both glutamine and glutamate levels and stable isotopic enrichments. Anal Biochem 147:92-102.

Deuschle K, Okumoto S, Fehr M, Looger LL, Kozhukh L, Frommer WB (2005) Construction and optimization of a family of genetically encoded metabolite sensors by semirational protein engineering. Protein Sci 14:2304-2314.

Dulla C, Tani H, Okumoto S, Frommer WB, Reimer RJ, Huguenard JR (2008) Imaging of glutamate in brain slices using FRET sensors. J Neurosci Methods 168:306-319.

Eid T, Thomas MJ, Spencer DD, Rundén-Pran E, Lai JC, Malthankar GV, Kim JH, Danbolt NC, Ottersen OP, de Lanerolle NC (2004) Loss of glutamine synthetase in the human epileptogenic hippocampus: possible mechanism for raised extracellular glutamate in mesial temporal lobe epilepsy. Lancet 363:28-37.

Eid T, Ghosh A, Wang Y, Beckström H, Zaveri HP, Lee TS, Lai JC, Malthankar-Phatak GH, de Lanerolle NC (2008) Recurrent seizures and brain pathology after inhibition of glutamine synthetase in the hippocampus in rats. Brain 131:2061-2070.

Erecińska M, Silver IA (1990) Metabolism and role of glutamate in mammalian brain. Prog Neurobiol 35:245-296.

Fonnum F (1993) Regulation of the synthesis of the transmitter glutamate pool. Prog Biophys Mol Biol 60:47-57.

Fricke MN, Jones-Davis DM, Mathews GC (2007) Glutamine uptake by system A transporters maintains neurotransmitter GABA synthesis and inhibitory synaptic transmission. J Neurochem 102:1895-1904.

Furness DN, Dehnes Y, Akhtar AQ, Rossi DJ, Hamann M, Grutle NJ, Gundersen V, Holmseth S, Lehre KP, Ullensvang K, Wojewodzic M, Zhou Y, Attwell D, Danbolt NC (2008) A quantitative assessment of glutamate uptake into hippocampal synaptic terminals and astrocytes: new insights into a neuronal role for excitatory amino acid transporter 2 (EAAT2). Neuroscience 157:80-94.

Geng MY, Saito H, Nishiyama N (1997) Protective effects of pyridoxal phosphate against glucose deprivation-induced damage in cultured hippocampal neurons. J Neurochem 68:2500-2506.

Gibbs ME, O’Dowd BS, Hertz L, Robinson SR, Sedman GL, Ng KT (1996) Inhibition of glutamine synthetase activity prevents memory consolidation. Brain Res Cogn Brain Res 4:57-64.

Giove F, Garreffa G, Peca S, Carní M, Macrì MA, Di Bonaventura C, Vaudano AE, Giallonardo AT, Prencipe M, Bozzao L, Pantano P, Colonnese C, Maraviglia B (2006) Metabolic alteration transients during paroxysmal activity in an epileptic patient with fixation-off sensitivity: a case study. Magn Reson Imaging 24:373-379.

Gutnick MJ, Connors BW, Prince DA (1982) Mechanisms of neocortical epileptogenesis in vitro. J Neurophysiol 48:1321-1335.

Hamberger AC, Chiang GH, Nylén ES, Scheff SW, Cotman CW (1979) Glutamate as a CNS transmitter. I. Evaluation of glucose and glutamine as precursors for the synthesis of preferentially released glutamate. Brain Res 168:513-530.

Henry PG, Russeth KP, Tkac I, Drewes LR, Andrews MT, Gruetter R (2007) Brain energy metabolism and neurotransmission at near-freezing temperatures: in vivo (1)H MRS study of a hibernating mammal. J Neurochem 101:1505-1515.

Hrabetová S, Nicholson C (2000) Dextran decreases extracellular tortuosity in thick-slice ischemia model. J Cereb Blood Flow Metab 20:1306-1310

Kam K, Nicoll R (2007) Excitatory synaptic transmission persists independently of the glutamate-glutamine cycle. J Neurosci 27:9192-9200.

Kanamori K, Ross BD (2006) Kinetics of glial glutamine efflux and the mechanism of neuronal uptake studied in vivo in mildly hyperammonemic rat brain. J Neurochem 99:1103-1113.

Kaneko T (2000) Enzymes responsible for glutamate synthesis and degradation. In: Handbook of chemical neuroanatomy: glutamate, Vol 18, Chap VII (Ottersen OP, Storm-Mathisen J, eds), pp 202-230. Amsterdam: Elsevier Science.

Kapetanovic IM, Yonekawa WD, Kupferberg HJ (1993) Time-related loss of glutamine from hippocampal slices and concomitant changes in neurotransmitter amino acids. J Neurochem 61:865-872.

Katz B (1962) The transmission of impulses from nerve to muscle, and the subcellular unit of synaptic action. Proc R Soc Lond B Biol Sci 155:455-477.

Katz B, Miledi R (1965) Release of acetylcholine from a nerve terminal by electric pulses of variable strength and duration. Nature 207: 1097-1098.

Katz B, Miledi R (1968) The role of calcium in neuromuscular facilitation. J Physiol 195:481-492.

Kvamme E (1998) Synthesis of glutamate and its regulation. Prog Brain Res 116:73-85.

Lamar C Jr, Sellinger OZ (1965) The inhibition in vivo of cerebral glutamine synthetase and glutamine transferase by the convulsant methionine sulfoximine. Biochem Pharmacol 14:489-506.

Lerma J, Herranz AS, Herreras O, Abraira V, Martín del Río R (1986) In vivo determination of extracellular concentration of amino acids in the rat hippocampus. A method based on brain dialysis and computerized analysis. Brain Res 384:145-155.

Liang SL, Carlson GC, Coulter DA (2006) Dynamic regulation of synaptic GABA release by the glutamate-glutamine cycle in hippocampal area CA1. J Neurosci 26:8537-8548.

Lieth E, LaNoue KF, Berkich DA, Xu B, Ratz M, Taylor C, Hutson SM (2001) Nitrogen shuttling between neurons and glial cells during glutamate synthesis. J Neurochem 76:1712-1723.

Mackenzie B, Erickson JD (2004) Sodium-coupled neutral amino acid (system N/A) transporters of the SLC38 gene family. Pflugers Arch 447:784-795.

Manning JM, Moore S, Rowe WB, Meister A (1969) Identification of L-methionine S-sulfoximine as the diastereoisomer of L-methionine SRsulfoximine that inhibits glutamine synthetase. Biochemistry 8:2681-2685.

Martinez-Hernandez A, Bell KP, Norenberg MD (1977) Glutamine synthetase: glial localization in brain. Science 195:1356-1358.

Masson J, Darmon M, Conjard A, Chuhma N, Ropert N, Thoby-Brisson M, Foutz AS, Parrot S, Miller GM, Jorisch R, Polan J, Hamon M, Hen R, Rayport S (2006) Mice lacking brain/kidney phosphate-activated glutaminase have impaired glutamatergic synaptic transmission, altered breathing, disorganized goal-directed behavior and die shortly after birth. J Neurosci 26:4660-4671.

McKenna MC (2007) The glutamate-glutamine cycle is not stoichiometric: fates of glutamate in brain. J Neurosci Res 85:3347-3358.

Mienville JM, Vicini S (1987) A pyridazinyl derivative of gammaaminobutyric acid (GABA), SR 95531, is a potent antagonist of Clchannel opening regulated by GABAA receptors. Neuropharmacology 26:779-783.

Min MY, Appenteng K (1996) Multimodal distribution of amplitudes of miniature and spontaneous EPSPs recorded in rat trigeminal motoneurones. J Physiol 494:171-182.

Otsuki T, Nakama H, Kanamatsu T, Tsukada Y (2005) Glutamate metabolism in epilepsy: 13C-magnetic resonance spectroscopy observation in the human brain. Neuroreport 16:2057-2060.

Oz G, Berkich DA, Henry PG, Xu Y, LaNoue K, Hutson SM, Gruetter R (2004) Neuroglial metabolism in the awake rat brain: $\mathrm{CO}_{2}$ fixation increases with brain activity. J Neurosci 24:11273-11279.

Pan JW, Williamson A, Cavus I, Hetherington HP, Zaveri H, Petroff OA, Spencer DD (2008) Neurometabolism in human epilepsy. Epilepsia 49 [Suppl 3]:31-41.

Prince DA (1966) Modification of focal cortical epileptogenic discharge by afferent influences. Epilepsia 7:181-201.

Pyle JL, Kavalali ET, Choi S, Tsien RW (1999) Visualization of synaptic activity in hippocampal slices with FM1-43 enabled by fluorescence quenching. Neuron 24:803-808.

Rae C, Hare N, Bubb WA, McEwan SR, Bröer A, McQuillan JA, Balcar VJ, Conigrave AD, Bröer S (2003) Inhibition of glutamine transport depletes glutamate and GABA neurotransmitter pools: further evidence for metabolic compartmentation. J Neurochem 85:503-514.

Rothman DL, Behar KL, Hyder F, Shulman RG (2003) In vivo NMR studies of the glutamate neurotransmitter flux and neuroenergetics: implications for brain function. Annu Rev Physiol 65:401-427.

Rothstein JD, Dykes-Hoberg M, Pardo CA, Bristol LA, Jin L, Kuncl RW, Kanai Y, Hediger MA, Wang Y, Schielke JP, Welty DF (1996) Knockout of glutamate transporters reveals a major role for astroglial transport in excitotoxicity and clearance of glutamate. Neuron 16:675-686.

Rowe WB, Meister A (1970) Identification of L-methionine-S-sulfoximine as the convulsant isomer of methionine sulfoximine. Proc Natl Acad Sci U S A 66:500-506.

Sandow N, Zahn RK, Gabriel S, Heinemann U, Lehmann TN (2009) Glutamine induces epileptiform discharges in superficial layers of the medial 
entorhinal cortex from pilocarpine-treated chronic epileptic rats in vitro. Epilepsia 50:849-858.

Shank RP, Bennett GS, Freytag SO, Campbell GL (1985) Pyruvate carboxylase: an astrocyte-specific enzyme implicated in the replenishment of amino acid neurotransmitter pools. Brain Res 329:364-367.

Sibson NR, Mason GF, Shen J, Cline GW, Herskovits AZ, Wall JE, Behar KL, Rothman DL, Shulman RG (2001) In vivo (13)C NMR measurement of neurotransmitter glutamate cycling, anaplerosis and TCA cycle flux in rat brain during. J Neurochem 76:975-989.

Su TZ, Campbell GW, Oxender DL (1997) Glutamine transport in cerebellar granule cells in culture. Brain Res 757:69-78.

Syková E, Nicholson C (2008) Diffusion in brain extracellular space. Physiol Rev 88:1277-1340.

Tamarappoo BK, Raizada MK, Kilberg MS (1997) Identification of a system
$\mathrm{N}$-like $\mathrm{Na}(+)$-dependent glutamine transport activity in rat brain neurons. J Neurochem 68:954-960.

Tanaka K, Watase K, Manabe T, Yamada K, Watanabe M, Takahashi K, Iwama H, Nishikawa T, Ichihara N, Kikuchi T, Okuyama S, Kawashima N, Hori S, Takimoto M, Wada K (1997) Epilepsy and exacerbation of brain injury in mice lacking the glutamate transporter GLT-1. Science 276:1699-1702.

Tani H, Bandrowski AE, Parada I, Wynn M, Huguenard JR, Prince DA, Reimer RJ (2007) Modulation of epileptiform activity by glutamine and system A transport in a model of post-traumatic epilepsy. Neurobiol Dis 25:230-238.

Wolosker H, de Souza DO, de Meis L (1996) Regulation of glutamate transport into synaptic vesicles by chloride and proton gradient. J Biol Chem 271:11726-11731. 